\title{
ATOMS : ALMA three-millimeter observations of massive star-forming regions - II. Compact objects in ACA observations and star formation scaling relations
}

\author{
Liu, Tie
}

2020-08

Liu , T , Evans , N J , Kim , K-T , Goldsmith , P F , Liu, S-Y, Zhang , Q, Tatematsu , K, Wang , K, Juvela , M , Bronfman , L , Cunningham , M R , Garay , G , Hirota , T, Lee , J-E , Kang , S-J , Li , D , Li , P-S , Mardones, D , Qin , S-L , Ristorcelli , I, Tej , A , Toth , L V , Wu , J-W , Wu , Y-F , Yi , H , Yun, H-S , Liu , H-L, Peng , Y-P , Li , J , Li , S H, Lee , C W , Shen, Z-Q, Baug , T , Wang , J-Z, Zhang, Y, Issac , N , Zhu , F-Y , Luo , Q-Y , Liu , X-C , Xu , F-W , Wang , Y, Zhang , C , Ren , Z \& Zhang , C 2020 , ' ATOMS : ALMA three-millimeter observations of massive star-forming regions - II. Compact objects in ACA observations and star formation scaling relations ' , Monthly Notices of the Royal Astronomical Society, vol. 496 , no. 3 , pp. 2821-2835 . https://doi.org/10.1093/mnras/staa1501

http://hdl.handle.net/10138/320607

https://doi.org/10.1093/mnras/staa1501

cc_by_nc_sa

publishedVersion

Downloaded from Helda, University of Helsinki institutional repository.

This is an electronic reprint of the original article.

This reprint may differ from the original in pagination and typographic detail.

Please cite the original version. 


\title{
ATOMS: ALMA three-millimeter observations of massive star-forming regions - II. Compact objects in ACA observations and star formation scaling relations
}

\section{Tie Liu ${ }^{\bullet},{ }^{1,2,3 \star}$ Neal J. Evans, ${ }^{2,4}$ Kee-Tae Kim ${ }^{\circledR},{ }^{2,5}$ Paul F. Goldsmith, ${ }^{6}$}

Sheng-Yuan Liu, ${ }^{7}$ Qizhou Zhang, ${ }^{8}$ Ken'ichi Tatematsu ${ }^{\odot},{ }^{9}$ Ke Wang, ${ }^{10}$ Mika Juvela, ${ }^{11}$ Leonardo Bronfman, ${ }^{12}$ Maria. R. Cunningham, ${ }^{13}$ Guido Garay, ${ }^{12}$ Tomoya Hirota ${ }^{\odot}, 9$ Jeong-Eun Lee, ${ }^{14}$ Sung-Ju Kang, ${ }^{2}$ Di Li ${ }^{\oplus},{ }^{15,16,17}$ Pak-Shing Li, ${ }^{18}$ Diego Mardones, ${ }^{12}$ Sheng-Li Qin, ${ }^{19}$ Isabelle Ristorcelli, ${ }^{20}$ Anandmayee Tej, ${ }^{21}$ L. Viktor Toth ${ }^{\oplus}, 2$ Jing-Wen Wu, ${ }^{15}$ Yue-Fang Wu, ${ }^{23}$ Hee-weon Yi, ${ }^{14}$ Hyeong-Sik Yun, ${ }^{14}$ Hong-Li Liu ${ }^{\oplus},{ }^{24}$ Ya-Ping Peng, ${ }^{25}$ Juan Li ${ }^{\oplus},{ }^{1,26}$ Shang Huo Li, ${ }^{1}$ Chang Won Lee, ${ }^{2,5}$ Zhi-Qiang Shen, ${ }^{1,26}$ Tapas Baug, ${ }^{10}$ Jun-Zhi Wang, ${ }^{1,26}$ Yong Zhang, ${ }^{27}$ Namitha Issac, ${ }^{21}$ Feng-Yao Zhu, ${ }^{1}$ Qiu-Yi Luo, ${ }^{1}$ Xun-Chuan Liu, ${ }^{23}$ Feng-Wei Xu, ${ }^{23}$ Yu Wang, ${ }^{23}$ Chao Zhang, ${ }^{19}$ Zhiyuan Ren ${ }^{15}$ and Chao Zhang ${ }^{15}$

Affiliations are listed at the end of the paper

Accepted 2020 May 25. Received 2020 May 19; in original form 2020 April 27

\begin{abstract}
We report studies of the relationships between the total bolometric luminosity ( $L_{\mathrm{bol}}$ or $L_{\mathrm{TIR}}$ ) and the molecular line luminosities of $J=1-0$ transitions of $\mathrm{H}^{13} \mathrm{CN}, \mathrm{H}^{13} \mathrm{CO}^{+}, \mathrm{HCN}$, and $\mathrm{HCO}^{+}$ with data obtained from ACA observations in the 'ATOMS' survey of 146 active Galactic starforming regions. The correlations between $L_{\mathrm{bol}}$ and molecular line luminosities $L_{\mathrm{mol}}^{\prime}$ of the four transitions all appear to be approximately linear. Line emission of isotopologues shows as large scatters in $L_{\mathrm{bol}}-L_{\mathrm{mol}}^{\prime}$ relations as their main line emission. The $\log \left(L_{\mathrm{bol}} / L_{\mathrm{mol}}^{\prime}\right)$ for different molecular line tracers have similar distributions. The $L_{\mathrm{bol}}$-to- $L_{\mathrm{mol}}^{\prime}$ ratios do not change with galactocentric distances $\left(R_{\mathrm{GC}}\right)$ and clump masses $\left(M_{\text {clump }}\right)$. The molecular line luminosity ratios (HCN-to-HCO ${ }^{+}, \mathrm{H}^{13} \mathrm{CN}$-to- $\mathrm{H}^{13} \mathrm{CO}^{+}$, $\mathrm{HCN}$-to- $\mathrm{H}^{13} \mathrm{CN}$, and $\mathrm{HCO}^{+}$-to- $\mathrm{H}^{13} \mathrm{CO}^{+}$) all appear constant against $L_{\mathrm{bol}}$, dust temperature ( $\left.T_{\mathrm{d}}\right), M_{\mathrm{clump}}$, and $R_{\mathrm{GC}}$. Our studies suggest that both the main lines and isotopologue lines are good tracers of the total masses of dense gas in Galactic molecular clumps. The large optical depths of main lines do not affect the interpretation of the slopes in star formation relations. We find that the mean star formation efficiency (SFE) of massive Galactic clumps in the 'ATOMS' survey is reasonably consistent with other measures of the SFE for dense gas, even those using very different tracers or examining very different spatial scales.
\end{abstract}

Key words: stars: formation - ISM: clouds - ISM: molecules - galaxies: star formation.

\section{INTRODUCTION}

Since the pioneering works by Gao \& Solomon (2004) and Wu et al. (2005), many observational studies towards external galaxies (Graciá-Carpio et al. 2006; Juneau et al. 2009; García-Burillo et al. 2012; Greve 2014; Zhang et al. 2014; Chen et al. 2015; Liu et al. 2015; Tan et al. 2018; Jiménez-Donaire et al. 2019) and Galactic molecular clouds (Wu et al. 2010; Liu et al. 2016; Stephens, Jackson \& Whitaker 2016) have revealed a strong linear relationship between the recent star formation rate (SFR), as traced by the total infrared emission, and the dense molecular gas mass that is indicated by line luminosities $\left(L_{\text {mol }}^{\prime}\right)$ of dense molecular gas tracers (e.g. $\mathrm{HCN}, \mathrm{HCO}^{+}$, and CS). This so-called 'dense gas 
star formation law' may imply that the dense molecular gas rather than the total molecular gas is the direct fuel for star formation (Kennicutt \& Evans 2012; Vutisalchavakul, Evans \& Heyer 2016). While significant progress has been made in recent years, the origin of this relationship is still under debate.

The $J=1-0$ transitions of $\mathrm{HCN}$ and $\mathrm{HCO}^{+}$are among the most commonly used tracers in the studies of 'dense gas star formation law'. However, the emission lines of these two dense gas tracers tend to be optically thick in dense parts of molecular clouds (e.g. Sanhueza et al. 2012; Hoq et al. 2013; Shimajiri et al. 2017). In the limit that all transitions are optically thin and only collisional excitation is important, but densities are far below the critical density, the emission would be proportional to $n \times n_{\text {mol }}$, where $n$ is the number density of colliders (mainly $\mathrm{H}_{2}$ ) and $n_{\text {mol }}$ is the density of the line-emitting molecules, thus proportional to $n^{2}$ for constant abundance. At the other extreme, the emission from an optically thick, thermalized line (e.g. ${ }^{12} \mathrm{CO}$ ) is not sensitive to density at all. While the extremes rarely apply, and the rich energy level structure of molecules permits more interesting excitation (masers, cooling of excitation temperatures below the temperature of the CMB, etc.), the above limiting cases illustrate why the emission from rare isotopologues is more highly weighted towards denser regions than their corresponding main lines. To capture some of the effects, the concept of effective excitation density was introduced to indicate the density needed to produce a line of a characteristic strength $\left(1 \mathrm{~K} \mathrm{~km} \mathrm{~s}^{-1}\right.$ ), given a column density typical for each species (Evans 1999; Shirley 2015). The low effective excitation densities of the two main transitions of $\mathrm{HCN}$ and $\mathrm{HCO}^{+}\left(4.5 \times 10^{3} \mathrm{~cm}^{-3}\right.$ for the $\mathrm{HCN} J=1-0$ line and $5.3 \times 10^{2} \mathrm{~cm}^{-3}$ for the $\mathrm{HCO}^{+} J=1-0$ line at $20 \mathrm{~K}$; Shirley 2015) indicate that they can be excited in low density $\left(n<10^{3} \mathrm{~cm}^{-3}\right)$ gas and are often optically thick.

Indeed, recent observations of nearby Giant molecular clouds (Kauffmann et al. 2017; Pety et al. 2017; Shimajiri et al. 2017) indicate that $\mathrm{HCN}(1-0)$ and $\mathrm{HCO}^{+}(1-0)$ are easily detected in extended translucent regions at a typical density of $500 \mathrm{~cm}^{-3}$ and are poor tracers of dense structures such as filaments or cores. Stephens et al. (2016) have argued that most of the Galaxy's luminosity of $\mathrm{HCN}$ may arise from distributed, sub-thermal emission rather than from dense gas. Most recently, Evans et al. (2020) also found that a substantial fraction (in most cases, the majority) of the total $\mathrm{HCN}$ (1-0) and $\mathrm{HCO}^{+}(1-0)$ line luminosity in six distant $(d \sim 3.5-$ $10.4 \mathrm{kpc}$ ) clouds arises in gas below the $A_{\mathrm{V}} \sim 8$ mag threshold, above which the vast majority of dense cores and YSOs are found in nearby clouds (Heiderman et al. 2010; Lada, Lombardi \& Alves 2010; Lada et al. 2012). These studies have challenged the ideas that these commonly used tracers (e.g. $J=1-0$ of $\mathrm{HCN}$ and $\mathrm{HCO}^{+}$) can reveal well the spatial distribution of star-forming gas in clouds.

As discussed above, in contrast to the main lines, emission from the isotopologues are optically thinner because of their much lower abundances. The effective densities of $\mathrm{H}^{13} \mathrm{CN} J=1-0$ and $\mathrm{H}^{13} \mathrm{CO}^{+} J=1-0$ at $20 \mathrm{~K}$ are about $1.6 \times 10^{5}$ and $2.2 \times 10^{4} \mathrm{~cm}^{-3}$, respectively, which are about 40 times higher than their main lines (Shirley 2015). Therefore, they can potentially be better tracers of the column densities and dense structures in molecular clouds (e.g. Pety et al. 2017; Shimajiri et al. 2017). The observations of these isotopologues can also help estimate the opacity of their corresponding main line transitions, enabling us to quantify how opacity affects the 'dense gas star formation law' with main lines. Studies of the 'dense gas star formation law' with isotopologues, however, are very rare. Stephens et al. (2016) used $\mathrm{H}^{13} \mathrm{CO}^{+} J=$ $1-0$ in their studies but they detected the $\mathrm{H}^{13} \mathrm{CO}^{+} J=1-0$ emission only in dozens of sources in their sample of $\sim 300$ clumps.
In addition, most stars form in gravitationally bound structures (clumps or cores) in molecular clouds. Therefore, it is also essential to evaluate how those lines (main lines and their isotopologues) trace the total gas mass of these gravitationally bound structures.

In this work, we investigate the 'dense gas star formation law' with $\mathrm{H}^{13} \mathrm{CN} J=1-0$ and $\mathrm{H}^{13} \mathrm{CO}^{+} J=1-0$ lines as well as their corresponding main lines towards a large sample of 146 Galactic star-forming clumps. By doing this, we evaluate the reliability of the main lines being used to trace the total dense gas mass.

This study is part of an ALMA survey program, the 'ALMA Three-millimeter Observations of Massive Star-forming regions' (ATOMS). The sample and observations of the 'ATOMS' survey are introduced in Liu et al. (2020). The 'ATOMS' observed a large sample of 146 active star-forming regions with IRAS colours characteristic of Ultra-Compact (UC) H II regions (Bronfman, Nyman \& May 1996). More than 90 per cent of the sources in the 'ATOMS' sample are potentially forming high-mass stars (Liu et al. 2020). In this paper, we only use the data from observations with the Atacama Compact $7 \mathrm{~m}$ Array (ACA; Morita Array). The ACA data are particularly useful for this study because they respond primarily to the scale of clumps ( $\gtrsim 0.2 \mathrm{pc})$, rather than cores $(\lesssim 0.1 \mathrm{pc})$. The dense gas star formation relations will surely break down at the level of cores, but will plausibly be relevant on the scale of clumps.

\section{OBSERVATIONS}

The ALMA observations of the 'ATOMS' survey have been summarized in Liu et al. (2020). We here briefly introduce the observations with the ACA. Observations with the ACA were conducted from late September to mid November in 2019 with band 3 (Project ID: 2019.1.00685.S; PI: Tie Liu). The IRAS names, phase centers (columns 2-3), systemic velocities (column 4), distances (column 5), and galactocentric distances (column 6) of the targeted sources are listed in Table A1. The typical ACA observing time per source is $\sim 8 \mathrm{~min}$. The angular resolution and maximum recovered angular scale (MRS) in ACA observations are $\sim 13.1-13.8$ arcsec and $\sim 53.8-76.2$ arcsec, respectively. The ACA observations are sensitive to angular scales smaller than $\sim 60$ arcsec but the MRS is larger than the angular sizes of most clumps in the sample (Faúndez et al. 2004). The $J=1-0$ transitions of $\mathrm{HCN}, \mathrm{HCO}^{+}, \mathrm{H}^{13} \mathrm{CN}$, and $\mathrm{H}^{13} \mathrm{CO}^{+}$are included in four spectral windows in the lower sideband. The spectral resolution for $\mathrm{HCN} J=1-0$ and $\mathrm{HCO}^{+} J=$ $1-0$ is $61.035 \mathrm{kHz}$ ( or $\sim 0.2 \mathrm{~km} \mathrm{~s}^{-1}$ ), while the spectral resolution for $\mathrm{H}^{13} \mathrm{CN} J=1-0$ and $\mathrm{H}^{13} \mathrm{CO}^{+} J=1-0$ is $0.122 \mathrm{MHz}$ (or $\sim 0.4 \mathrm{~km} \mathrm{~s}^{-1}$ ). Calibration and imaging were carried out using the CASA software package version 5.6 (McMullin et al. 2007). The continuum visibility data are constructed using the line-free spectral channels. All images are primary beam corrected. The achieved sensitivity of the ACA observations is around 50-90 $\mathrm{mJy}^{\text {beam }}{ }^{-1}$ per $0.122 \mathrm{MHz}$ channel for lines.

\section{RESULTS}

\subsection{Compact objects}

We extracted compact objects from $3 \mathrm{~mm}$ continuum emission maps and the integrated intensity maps of the four molecular lines. Fig. 1 shows the $3 \mathrm{~mm}$ continuum emission and integrated intensity maps for three sources. The compact objects in $3 \mathrm{~mm}$ continuum emission and molecular line emission can be easily identified by eye. In total, we detected 173, 184, 190, 189, and 182 compact sources from 

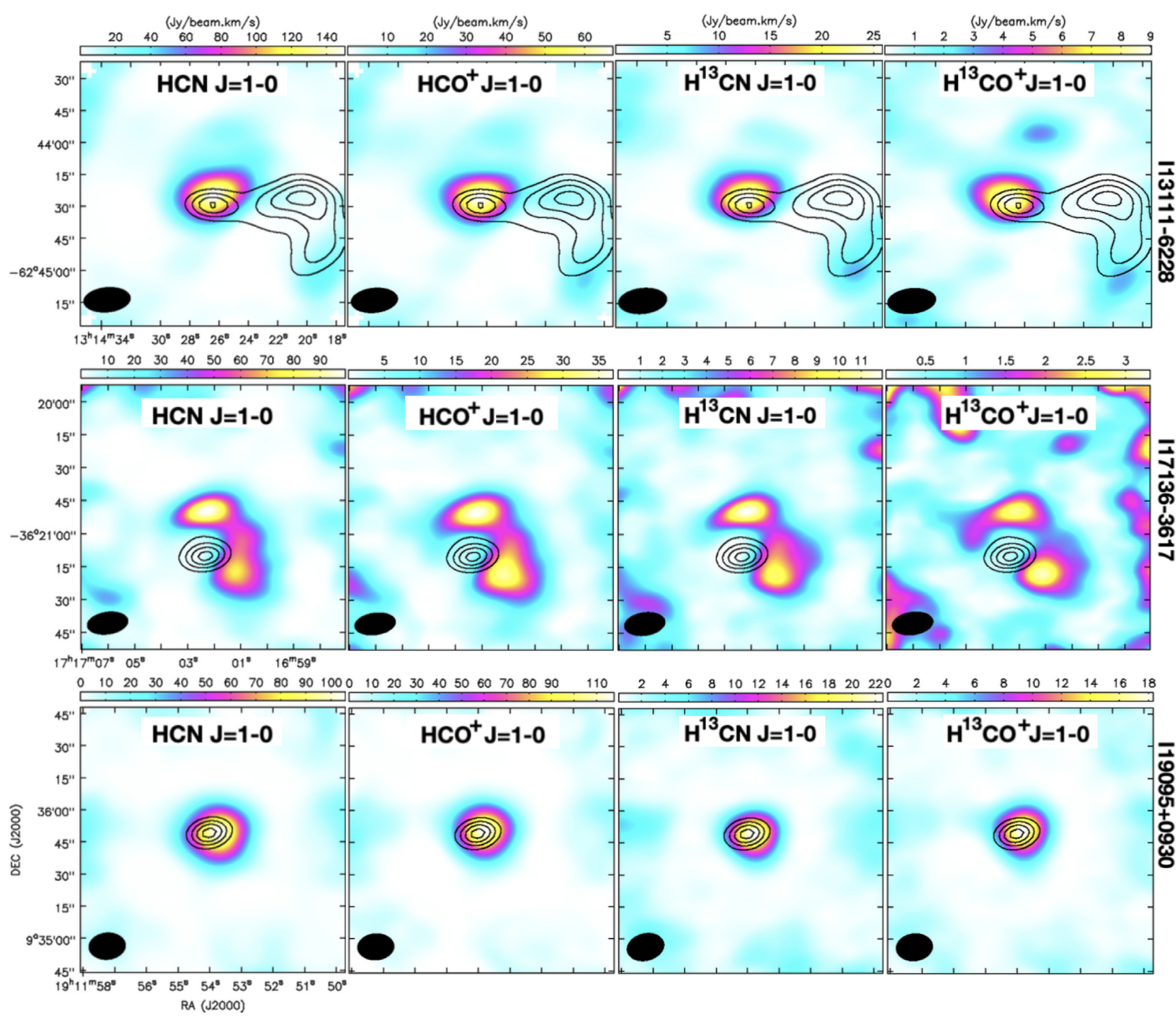

Figure 1. Integrated intensity maps of four molecular lines are shown as colour-scale images for three example sources. I13111-6228, I17136-3617, and I19095+0930 are shown in upper, middle, and lower panels, respectively. The $3 \mathrm{~mm}$ continuum is shown in contours. The contour levels are from 30 per cent to 90 per cent in steps of 20 per cent of peak values. The peak values of $3 \mathrm{~mm}$ continuum emission for I13111-6228, I17136-3617, and I19095+0930 are 0.088, 1.814 , and $0.307 \mathrm{Jy}$ beam $^{-1}$, respectively.

$3 \mathrm{~mm}$ continuum emission, $\mathrm{H}^{13} \mathrm{CN}, \mathrm{H}^{13} \mathrm{CO}^{+}, \mathrm{HCN}$, and $\mathrm{HCO}^{+}$ line emission, respectively.

As shown in Fig. 1 for I13111-6228, we identified multiple compact objects in $3 \mathrm{~mm}$ continuum in 27 targets. Some targeted sources also contain multiple objects in molecular line emission as shown in Fig. 1 for I17136-3617. We identified multiple objects in $30,32,35$, and 27 sources from $\mathrm{H}^{13} \mathrm{CN}, \mathrm{H}^{13} \mathrm{CO}^{+}, \mathrm{HCN}$, and $\mathrm{HCO}^{+}$integrated intensity maps, respectively. However, the majority $(\sim 80$ per cent) of sources contain only a single compact object in $3 \mathrm{~mm}$ continuum emission and in molecular line emission as I19095+0930 in Fig. 1.

From 2D Gaussian fits, we derived the relative coordinates (or offsets), aspect ratio values, effective radii $\left(R_{\text {eff }}\right)$, offsets from brightest continuum emission peaks $\left(d_{\text {peak }}\right)$, peak integrated intensity $\left(S_{\text {peak }}\right)$, and total integrated intensity $\left(S_{\text {total }}\right)$ for each compact object. The aspect ratio value is defined as the ratio between deconvolved FWHM major dimension $(a)$ and minor dimension $(b)$, and $R_{\text {eff }}$ is defined as $R_{\text {eff }}=\sqrt{a b}$. For compact objects identified in $\mathrm{H}^{13} \mathrm{CO}^{+} J=1-0$ and $\mathrm{HCO}^{+} J=1-0$ line emission, we also derived the source-averaged velocity $\left(V_{\mathrm{lsr}}\right)$ and velocity dispersion $(\sigma)$ from their corresponding Moment 1 and Moment 2 maps. All of these parameters for compact objects that are identified in $3 \mathrm{~mm}$ continuum, $\mathrm{H}^{13} \mathrm{CO}^{+}, \mathrm{H}^{13} \mathrm{CN}, \mathrm{HCO}^{+}$, and $\mathrm{HCN}$ are summerized in Table A1, Table A2, Table A3, Table A4 and Table A5, respectively.

Fig. 2 presents histograms of parameters $\left(S_{\text {peak }}, S_{\text {total }}\right.$, aspect ratio, and $R_{\text {eff }}$ ) for compact sources in $3 \mathrm{~mm}$ continuum emission. The median values for $S_{\text {peak }}$ and $S_{\text {total }}$ are $0.12 \mathrm{Jy}$ beam $^{-1}$ and $0.20 \mathrm{Jy}$, respectively. The median value of the aspect ratio is $\sim 1.6$. Among the 173 compact objects identified in $3 \mathrm{~mm}$ continuum emission, only 18 are with aspect ratio larger than 3 . The $R_{\text {eff }}$ ranges from 0.01 to $0.94 \mathrm{pc}$ with a median value of $0.18 \mathrm{pc}$. There are 45 compact objects that would be classified as dense cores with $R_{\text {eff }}$ smaller than $0.1 \mathrm{pc}$. There are 14 compact objects in distant sources having $R_{\text {eff }}$ larger than $0.5 \mathrm{pc}$. The detailed properties of those 

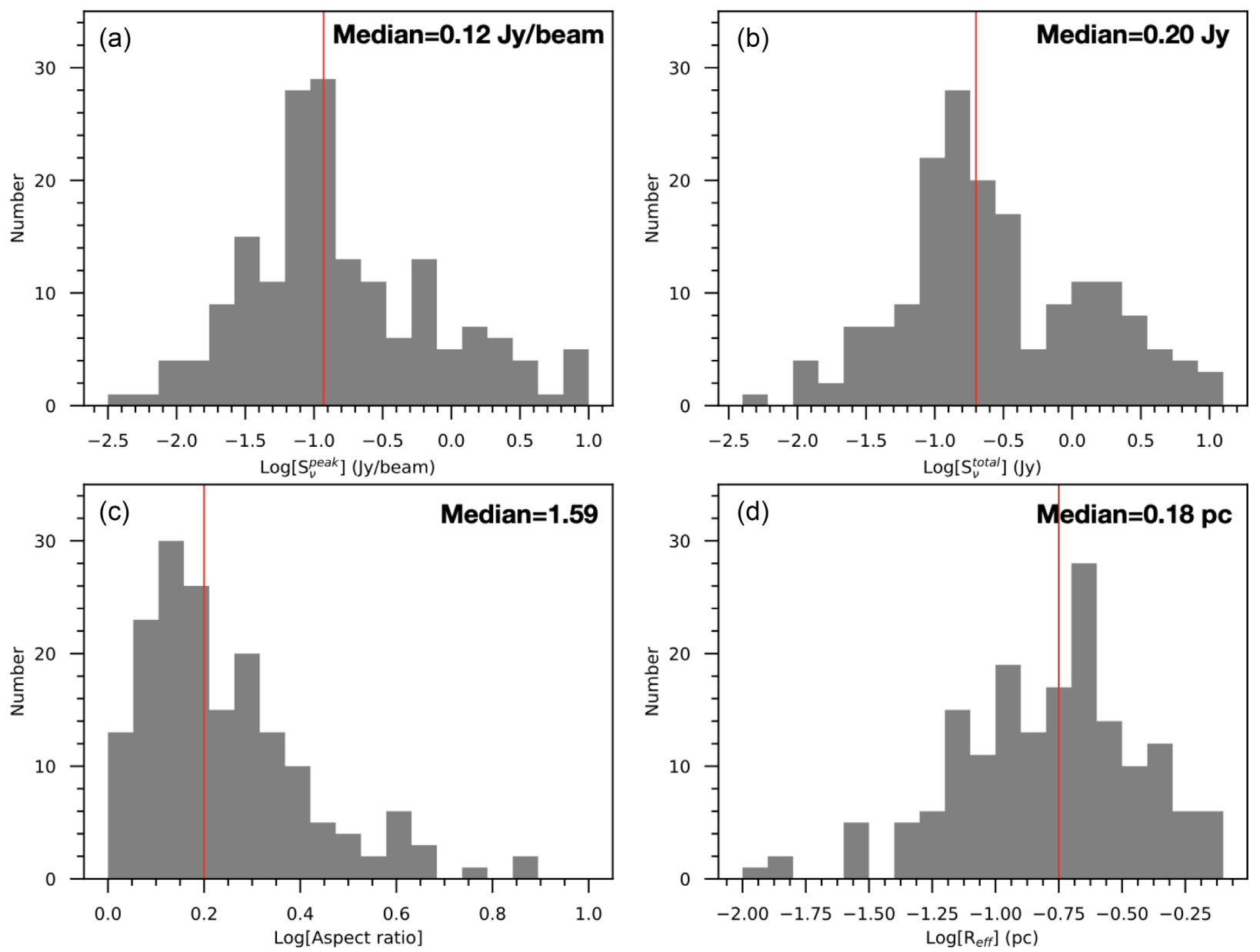

Figure 2. Histograms of parameters for the compact objects identified in $3 \mathrm{~mm}$ continuum emission. (a) peak flux density; (b) total flux density; (c) aspect ratios; (d) effective radii. The red solid lines are median values.

continuum objects will be discussed in forthcoming papers. The 3-mm continuum emission can have a significant contribution from free-free emission, compromising our ability to determine masses from the dust continuum emission. We use instead the literature values for the size $\left(\mathrm{R}_{\mathrm{eff}}\right)$, mass $\left(\mathrm{M}_{\mathrm{clump}}\right)$, etc. of the clumps (see Table A6). These are taken from single-dish maps at $0.87 \mathrm{~mm}$ (Urquhart, König \& Giannetti 2018) or $1.2 \mathrm{~mm}$ (Faúndez et al. 2004), minimizing the contamination by free-free emission.

In Fig. 3 , we compare the distributions of $R_{\text {eff }}$ for compact objects identified in $3 \mathrm{~mm}$ continuum emission and molecular line emission. The data are plotted with a Gaussian kernel density estimate using the function 'kdeplot' in the PYTHON package SEABORN. ${ }^{1}$ The median radii for compact objects in $3 \mathrm{~mm}$ continuum, $\mathrm{H}^{13} \mathrm{CN}$, $\mathrm{H}^{13} \mathrm{CO}^{+} \mathrm{HCN}$, and $\mathrm{HCO}^{+}$line emission are $0.18,0.25,0.28$, 0.33 , and $0.34 \mathrm{pc}$, respectively. Compact objects in $3 \mathrm{~mm}$ continuum emission have statistically smaller $R_{\text {eff }}$ than that of compact objects identified in molecular line emission. $R_{\text {eff }}$ for compact objects identified in $\mathrm{H}^{13} \mathrm{CN}$ and $\mathrm{H}^{13} \mathrm{CO}^{+}$line emission are also statistically smaller than compact objects identified in $\mathrm{HCN}$ and $\mathrm{HCO}^{+}$line emission. Interestingly, there seems to be a trend between $R_{\text {eff }}$ and effective excitation density for the lines. The higher the effective excitation density, the smaller the $R_{\text {eff }}$. These results are consistent

${ }^{1}$ https://seaborn.pydata.org/generated/seaborn.kdeplot.html

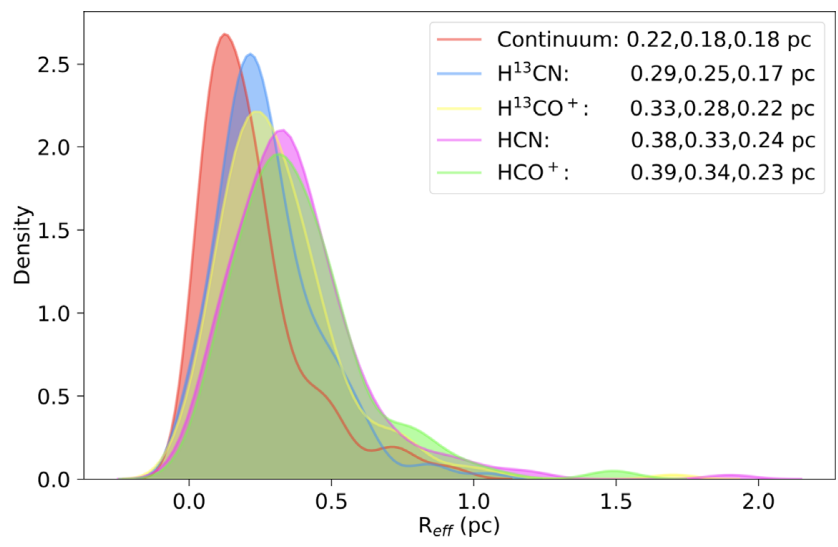

Figure 3. Distribution of $R_{\text {eff }}$ for compact objects identified in $3 \mathrm{~mm}$ continuum emission and molecular line emission, which are plotted using the function 'kdeplot' in the PYTHON package SEABORN. The numbers in the upper right box are mean, median, and standard deviation values.

with the idea that lines with higher effective excitation density trace denser and more compact regions of molecular clouds.

In Fig. 4(a), we compare the effective radii $\left(R_{\mathrm{ACA}}\right)$ of compact sources in ACA $3 \mathrm{~mm}$ continuum emission with the effective radii $\left(R_{\mathrm{SD}}\right)$ of their natal clumps derived in single dish observations. $R_{\mathrm{ACA}}$ is linearly correlated with $R_{\mathrm{SD}}$ as expected. Since the ACA is 

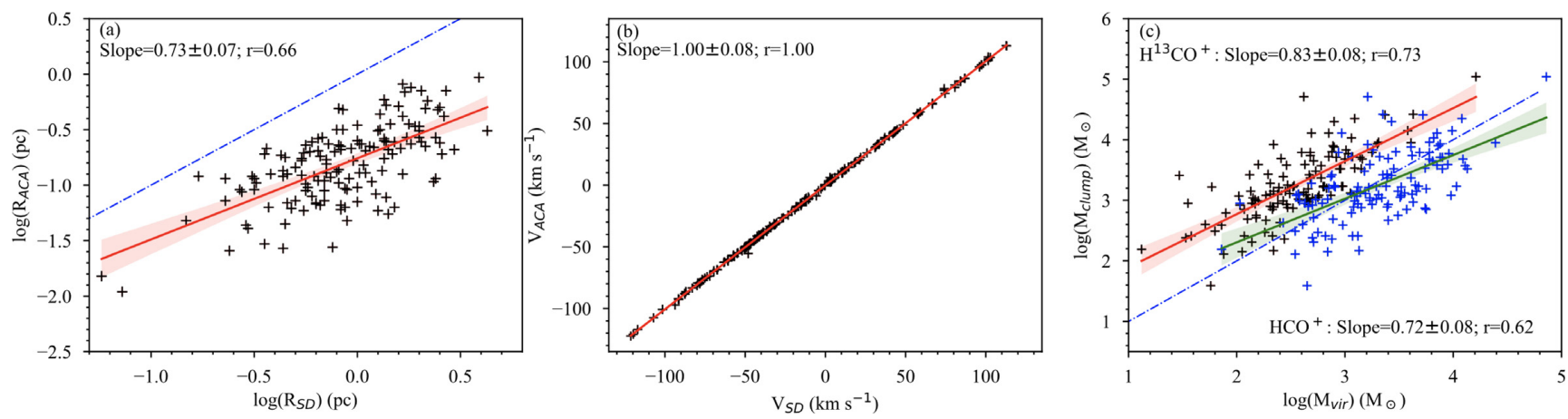

Figure 4. (a) Comparison of effective radii for compact objects in ACA $3 \mathrm{~mm}$ continuum observations and effective radii of whole clumps in single dish observations. The blue-dashed line represents that the two radii equal to each other. (b) Comparison of systemic velocities in ACA observations and single-dish observations. (c) Comparison of virial masses for compact objects identified in $\mathrm{H}^{13} \mathrm{CO}^{+}$(the black crosses) and $\mathrm{HCO}^{+}$(the blue crosses) line emission with total clump masses from single-dish observations. The red and green lines are linear fits. The blue-dashed line shows virial masses equaling to clump masses.

resolving out the more extended emission, $R_{\mathrm{ACA}}$ is systematically smaller than $R_{\mathrm{SD}}$. As shown in Fig. 4(b), the velocities derived from $\mathrm{H}^{13} \mathrm{CO}^{+} J=1-0$ line emission for compact sources are very consistent with the systemic velocities of their natal clumps from single dish CS $J=2-1$ line observations (Bronfman et al. 1996).

In some sources such as I17136-3617 shown in Fig. 1, gas emission peaks are clearly offset from $3 \mathrm{~mm}$ continuum emission peaks by one beam size. These sources may be highly evolved $\mathrm{H}$ II regions that have dispersed their natal gas clumps. In further analysis, we only focus on 119 sources, for which their gas emission and $3 \mathrm{~mm}$ continuum emission coincide well with each other (with separation smaller than one beam size of $\sim 15 \operatorname{arcsec}$ ). This will help reduce the evolutionary effect in 'dense gas star formation law' studies.

\subsection{Virial masses}

We derived virial masses for compact objects identified in $\mathrm{H}^{13} \mathrm{CO}^{+}$ and $\mathrm{HCO}^{+}$line emission following Bertoldi \& McKee (1992) and Wu et al. (2010). A correction $\left(a_{2} \sim 0.9\right)$ for a non-spherical shape in virial analysis is adopted for compact objects with aspect ratios larger than 3 (Bertoldi \& McKee 1992). For the others with smaller aspect ratios, this correction is negligible and has been omitted. We did not derive virial masses for compact objects identified in $\mathrm{H}^{13} \mathrm{CN}$ or $\mathrm{HCN}$ because of complications caused by hyperfine structure. The virial masses for objects identified in $\mathrm{HCO}^{+}$are probably overestimated because of line broadening of $\mathrm{HCO}^{+}$resulting from the high optical depth. $\mathrm{HCO}^{+}$also covers a larger region than $\mathrm{H}^{13} \mathrm{CO}^{+}$due to its lower effective excitation temperature (see Fig. 3).

The virial masses $\left(M_{\text {vir }}^{13}\right.$ and $\left.M_{\text {vir }}^{12}\right)$ derived from $\mathrm{H}^{13} \mathrm{CO}^{+}$and $\mathrm{HCO}^{+}$line emission are listed in the last two columns of Table A6. If there are more than one compact objects identified in a source, we simply sum the virial masses of the contributing objects. The virial masses derived from $\mathrm{H}^{13} \mathrm{CO}^{+}$range from 13 to $16260 M_{\odot}$ with a median value of $334 M_{\odot}$. About 90 per cent of the sources have virial masses larger than $100 M_{\odot}$. The virial masses for the main isotope are considerably larger than those based on the rare isotope. While the $\mathrm{HCO}^{+}$lines are generally optically thick, the other effect is that they extend over a larger region due to much lower effective excitation density. This leads to higher virial masses for $\mathrm{HCO}^{+}$.
In Fig. 4(c), we compare the virial masses with the total clump masses from single-dish measurements (Faúndez et al. 2004; Urquhart et al. 2018). The virial masses are strongly correlated with the total clump masses $\left(M_{\text {clump }}\right)$. The virial masses from $\mathrm{H}^{13} \mathrm{CO}^{+}$are systematically smaller than the total masses of their natal clumps with a median virial parameter $\left(\alpha=M_{\text {vir }} / M_{\text {clump }}\right.$ ) of $\sim 0.2$. The small value probably reflects the fact that the regions probed by $\mathrm{H}^{13} \mathrm{CO}^{+}$are smaller than those used to obtain the clump mass from the single-dish data. The virial masses from $\mathrm{HCO}^{+}$are comparable to the total clump masses, with a median virial parameter of $\sim 1.2$, indicating that $\mathrm{ACA}$ observations of $\mathrm{HCO}^{+}$trace structures similar to those traced by the single-dish millimeter continuum data, which are most likely gravitationally bound.

Small virial parameters have also been reported in many recent measurements of the thermodynamic properties in high-mass starforming regions (Pillai et al. 2011; Kauffmann, Pillai \& Goldsmith 2013; Zhang et al. 2015; Hull \& Zhang 2019), which appear to challenge the picture of star formation in which gas evolves in a state of equilibrium (e.g. Hull \& Zhang 2019). However, in this work and most of previous works magnetic field support were not taken into account in virial analysis. Magnetic support could be comparable to the turbulent and thermal support in high-mass star-forming regions, which can bring the dense gas close to a state of equilibrium (Pillai et al. 2011; Zhang et al. 2015; Sanhueza, Jackson \& Zhang 2017; Liu et al. 2018a,b; Hull \& Zhang 2019; Soam, Liu \& Andersson 2019; Tang et al. 2019). More detailed energetics comparison of the gravitational potential energy, turbulent support, thermal pressure, and magnetic support are needed in future analysis of virial equilibrium.

\subsection{The relations between infrared luminosities and molecular line luminosities}

In this section, we investigate how the infrared luminosities correlate with molecular line luminosities. Liu et al. (2016) has demonstrated that the bolometric luminosities $\left(L_{\mathrm{bol}}\right)$ from SED fits are nearly identical to the total infrared luminosities $\left(L_{\mathrm{TIR}}\right)$ for this 'ATOMS' sample. In this study, we still use $L_{\mathrm{bol}}$ (from Faúndez et al. 2004; Urquhart et al. 2018) instead of $L_{\mathrm{TIR}}$. The molecular line luminosities $\left(L_{\text {mol }}^{\prime}\right)$ are derived following Solomon et al. (1997) with the total integrated flux density. The $L_{\mathrm{bol}}$ and $L_{\text {mol }}^{\prime}$ are listed in Table A6. The statistics such as minimum, maximum, mean, 
median, and standard deviation of the logarithmic values of $L_{\mathrm{bol}}$, $L_{\text {mol }}^{\prime}$, and their ratios are summarized in Table 1.

Fig. 5 shows the correlations between $L_{\mathrm{bol}}$ and other parameters ( $M_{\text {clump }}, M_{\text {vir }}$ derived from $\mathrm{H}^{13} \mathrm{CO}^{+}$, and $L_{\text {mol }}^{\prime}$ ). All the relationships appear approximately linear. The correlations are fitted with a linear function using the function 'regplot' in the PYTHON package SEABORN. ${ }^{2}$ The slopes $(a)$, intercepts $(b)$ and correlation coefficients $(r)$ from linear regressions are summarized in Table 2. Since the effective excitation densities of isotopologue lines are significantly larger than their main lines (Shirley 2015), some models (e.g. Narayanan et al. 2008) would predict that the correlations between $L_{\mathrm{bol}}$ and $L_{\mathrm{mol}}^{\prime}$ are steeper for isotopologues than that for their main lines. In fact, the relations for isotopologues are not steeper and not even tighter for the 'ATOMS' sample (see Fig. 5).

The mean $\log \left(L_{\mathrm{bol}} / L_{\text {mol }}^{\prime}\right)$ ratios are $4.38,4.49,3.67$, and 3.86 for $\mathrm{H}^{13} \mathrm{CN}, \mathrm{H}^{13} \mathrm{CO}^{+}, \mathrm{HCN}$ and $\mathrm{HCO}^{+}$, respectively. The $L_{\mathrm{bol}}$-to$L_{\mathrm{HCN}}^{\prime}$ and $L_{\mathrm{bol}}$-to- $L_{\mathrm{HCO}^{+}}^{\prime}$ ratios for compact objects in the 'ATOMS' sample seem to be a factor of $\sim 5$ larger than the corresponding ratios for Galactic clumps (e.g. Wu et al. 2010; Stephens et al. 2016) and external galaxies (e.g. Jiménez-Donaire et al. 2019) measured with single dishes. This difference is also understandable because the line emission in our ACA observations is mainly from compact and dense structures (likely to be more gravitationally bound objects). In contrast, line emission in other works with single dishes also includes extended gas emission or even sub-thermal emission.

In Fig. 6, we plot the $L_{\mathrm{TIR}}$ (or $L_{\mathrm{bol}}$ ) as a function of the $L_{\mathrm{HCN}}^{\prime}$ for the 'ATOMS' measurements and literature measurements compiled by Jiménez-Donaire et al. (2019) . The literature measurements include single-dish observations towards both Galactic clumps and external galaxies. This scaling relation between $L_{\mathrm{TIR}}$ and $L_{\mathrm{HCN}}^{\prime}$ is nearly linear spanning almost 10 orders of magnitude in infrared and HCN luminosity. The data points in the 'ATOMS' measurements are clearly located above the relation determined by data points from single-dish measurements alone, indicating that the ACA observations in the 'ATOMS' survey may reveal structures with higher star formation efficiency (SFE) in clouds. These structures in the ACA observations are much denser and more gravitationally bound than those revealed in single dish measurements (see Fig. 4c).

\section{DISCUSSION}

\subsection{The dispersion in the $L_{\text {bol }}$-to- $L_{\text {mol }}^{\prime}$ ratios}

We noticed a significant scatter in $L_{\mathrm{bol}}$-to- $L_{\text {mol }}^{\prime}$ ratios in our data as shown in Fig. 7. The standard deviations in distributions of $\log \left(L_{\mathrm{bol}} / L_{\mathrm{mol}}^{\prime}\right)$ are around 0.5 for all four molecular line transitions. The scatters in distributions for isotopologues are as large as for their main lines. We test whether the distributions of $\log \left(L_{\mathrm{bol}} / L_{\text {mol }}^{\prime}\right)$ follow a normal distribution with Kolmogorov-Sminov test. ${ }^{3}$ The null hypothesis is that the distribution of $\log \left(L_{\mathrm{bol}} / L_{\text {mol }}^{\prime}\right)$ follows a normal distribution. The tests retain the null hypothesis with asymptotic significances (or P-values; 2-tailed) $\gtrsim 0.4$ for the four lines (see Fig. 7), indicating that the distributions of $\log \left(L_{\mathrm{bol}} / L_{\text {mol }}^{\prime}\right)$ likely follow a normal distribution. Stephens et al. (2016) also reported a significant scatter in $L_{\mathrm{bol}} / L_{\mathrm{HCN}}^{\prime}$ at $1 \mathrm{pc}$ clump-scale. They found that the difference between $L_{\mathrm{bol}} / L_{\mathrm{HCN}}^{\prime}$ for the lowest 10 per cent quantile

\footnotetext{
${ }^{2}$ https://seaborn.pydata.org/generated/seaborn.regplot.html

${ }^{3}$ https://www.spss-tutorials.com/spss-kolmogorov-smirnov-test-for-norm ality/
}

and the highest 90 per cent quantile is approximately two orders of magnitude. In our data, the difference is smaller, approximately one order of magnitude. It may imply that this ratio does not vary too much in gravitationally bound structures within clumps.

We have also investigated the similarities of distributions of $\log \left(L_{\mathrm{bol}} / L_{\mathrm{mol}}^{\prime}\right)$ for different molecular line tracers. In Table 3 , we test whether the $\log \left(L_{\mathrm{bol}} / L_{\text {mol }}^{\prime}\right)$ values for two molecular lines could be drawn from the same distribution with Kolmogorov-Sminov test after shifting the distributions by their mean values. The null hypothesis in tests is the shapes of the distributions for $\log \left(L_{\mathrm{bol}} / L_{\mathrm{mol}}^{\prime}\right)$ values are the same for the two molecular lines. With non-parametric tests, we found that the distributions of $\log \left(L_{\mathrm{bol}} / L_{\text {mol }}^{\prime}\right)$ for different molecular line tracers are very similar. In particular, the distributions of $\log \left(L_{\mathrm{bol}} / L_{\mathrm{HCN}}^{\prime}\right)$ and $\log \left(L_{\mathrm{bol}} / L_{\mathrm{HCO}^{+}}^{\prime}\right)$ show the highest similarity with asymptotic significances $\sim 0.97$ in Kolmogorov-Sminov test. The distributions for isotopologues $\left(\mathrm{H}^{13} \mathrm{CN}\right.$ and $\left.\mathrm{H}^{13} \mathrm{CO}^{+}\right)$are also similar to the distributions for their corresponding main lines ( $\mathrm{HCN}$ and $\mathrm{HCO}^{+}$) with asymptotic significance as high as $\sim 0.95$ in Kolmogorov-Sminov test. This implies that although the main lines may not trace well the dense structures (cores or filaments) within clumps/clouds (e.g. Kauffmann et al. 2017; Pety et al. 2017; Shimajiri et al. 2017; Liu et al. 2020), they can still reveal the total dense gas masses as well as their isotopologues in statistics.

Panels (a)-(d) in Fig. 8 present correlations between the $L_{\text {bol-to- }}$ $L_{\text {mol }}^{\prime}$ ratios and $L_{\text {bol }}$. Wu et al. $(2005,2010)$ suggest that above an infrared luminosity threshold of $\sim 10^{4.5} \mathrm{~L}_{\odot}$, the $L_{\text {bol }}$-to- $L_{\text {mol }}^{\prime}$ ratios become constant. However, we do not see such threshold in our data. There seems to be a clear trend that $L_{\mathrm{bol}}$-to- $L_{\text {mol }}^{\prime}$ ratio increases with $L_{\mathrm{bol}}$, spanning four orders of magnitude in $L_{\mathrm{bol}}$. This is likely caused by evolutionary effects of the sources, as suggested by Liu et al. (2016) and Stephens et al. (2016). Liu et al. (2016) reported a bimodal behaviour in the $L_{\mathrm{bol}}$-to- $L_{\text {mol }}^{\prime}$ correlations for clumps with different dust temperature, luminosity-to-mass ratio, and virial parameter. More luminous (or more evolved) sources seem to have consumed more gas, leading to higher $L_{\mathrm{bol}}$-to- $L_{\mathrm{mol}}^{\prime}$ ratios.

More evolved star-forming regions might have a higher dust temperature. To test the evolutionary effect, we present in Fig. 9 correlations between the $L_{\mathrm{bol}}$-to- $L_{\mathrm{mol}}^{\prime}$ ratios and dust temperature $T_{\mathrm{d}}$. As expected, there is a clear trend that $L_{\mathrm{bol}}$-to- $L_{\text {mol }}^{\prime}$ increases with $\mathrm{T}_{d}$. The trend is even more clearly seen for isotopologues. Same trend is seen in Fig. 8(a-d) as well where the trend is also more clearly seen in isotopologues.

Clump masses are not as sensitive to the evolutionary effect as bolometric luminosity or dust temperature. Panels (a)-(d) in Fig. 10 present correlations between the $L_{\text {bol }}$-to- $L_{\text {mol }}^{\prime}$ ratios and $M_{\text {clump }}$. Indeed, there is no clear trend between $L_{\mathrm{bol}}$-to- $L_{\text {mol }}^{\prime}$ ratios and $M_{\text {clump }}$ that spans three orders of magnitude in $M_{\text {clump }}$.

Panels (a)-(d) in Fig. 11 present correlations between the $L_{\text {bol }}$-to$L_{\mathrm{mol}}^{\prime}$ ratios and galactocentric distances $\left(R_{\mathrm{GC}}\right)$ for different tracers. Jiménez-Donaire et al. (2019) found that $L_{\mathrm{TIR}}$-to- $L_{\mathrm{HCN}}^{\prime}$ tends to increase with increasing $R_{\mathrm{GC}}$ in most of their targeted external galaxies, but with large galaxy-to-galaxy scatter. In our data, however, there is no clear trend for $L_{\mathrm{bol}}$-to- $L_{\mathrm{mol}}^{\prime}$ ratios against $R_{\mathrm{GC}}$, suggesting that this ratio may be constant for gravitationally bound clumps in different Galactic environments.

\subsection{Variations of molecular line luminosity ratios}

Fig. 12 plots the density distributions of various molecular line luminosity ratios (or integrated intensity ratios). Interestingly, we found that the distribution of $\mathrm{HCN}$-to- $\mathrm{HCO}^{+}$ratios is very similar to the distribution of $\mathrm{H}^{13} \mathrm{CN}$-to- $\mathrm{H}^{13} \mathrm{CO}^{+}$ratios. The non-parametric 
Table 1. Statistics of parameters of the 119 sources.

\begin{tabular}{lccccc}
\hline Parameters & Minimum & Maximum & Mean & Median & Std. deviation \\
\hline$R_{\text {eff }}$ & 0.15 & 4.26 & 1.09 & 0.84 & 0.75 \\
$T_{\mathrm{d}}$ & 19 & 46 & 29 & 29 & 5 \\
$L_{\mathrm{bol}}$ & 3.02 & 6.91 & 4.75 & 4.76 & 0.77 \\
$M_{\text {clump }}$ & 1.59 & 5.04 & 3.24 & 3.19 & 0.58 \\
$M_{\mathrm{vir}}^{13}$ & 1.12 & 4.21 & 2.53 & 2.52 & 0.50 \\
$M_{\mathrm{vir}}^{12}$ & 1.86 & 4.86 & 3.30 & 3.31 & 0.49 \\
$L_{\mathrm{H}^{13} \mathrm{CN}}^{\prime}$ & -1.08 & 1.75 & 0.37 & 0.31 & 0.57 \\
$L_{\mathrm{H}^{13} \mathrm{CO}^{+}}^{\prime}$ & -1.34 & 1.87 & 0.16 & 0.14 & 0.57 \\
$L_{\mathrm{HCN}}^{\prime}$ & -0.24 & 2.75 & 1.07 & 1.03 & 0.57 \\
$L_{\mathrm{HCO}}^{\prime}$ & -0.62 & 2.87 & 0.89 & 0.89 & 0.60 \\
$L_{\mathrm{bol}} / L_{\mathrm{H}^{13}}^{\prime}$ & 3.43 & 6.63 & 4.38 & 4.32 & 0.50 \\
$L_{\mathrm{bol}} / L_{\mathrm{H}^{13}}^{\prime} \mathrm{CO}^{+}$ & 3.26 & 6.53 & 4.59 & 4.57 & 0.52 \\
$L_{\mathrm{bol}} / L_{\mathrm{HCN}}^{\prime}$ & 2.47 & 5.53 & 3.67 & 3.63 & 0.53 \\
$L_{\mathrm{bol}}^{\prime} / L_{\mathrm{HCO}}^{\prime}$ & 2.43 & 5.57 & 3.86 & 3.80 & 0.53 \\
$L_{\mathrm{HCN}}^{\prime} / L_{\mathrm{HCO}}^{\prime}$ & -0.24 & 0.74 & 0.18 & 0.20 & 0.18 \\
$L_{\mathrm{HCN}}^{\prime} / L_{\mathrm{H}^{13}}^{\prime} \mathrm{CN}^{\prime}$ & -0.14 & 1.18 & 0.70 & 0.75 & 0.27 \\
$L_{\mathrm{HCO}}^{\prime} / L_{\mathrm{H}^{13}}$ & -0.03 & 1.28 & 0.73 & 0.77 & 0.26 \\
$L_{\mathrm{H}^{13} \mathrm{CN}^{+}}^{\prime} / L_{\mathrm{H}^{13}}^{\prime} \mathrm{CO}^{+}$ & -0.34 & 0.69 & 0.21 & 0.22 & 0.20 \\
\hline
\end{tabular}

${ }^{a}$ Except for $R_{\text {eff }}$ and $T_{\mathrm{d}}$, the other parameters are logarithmic values.
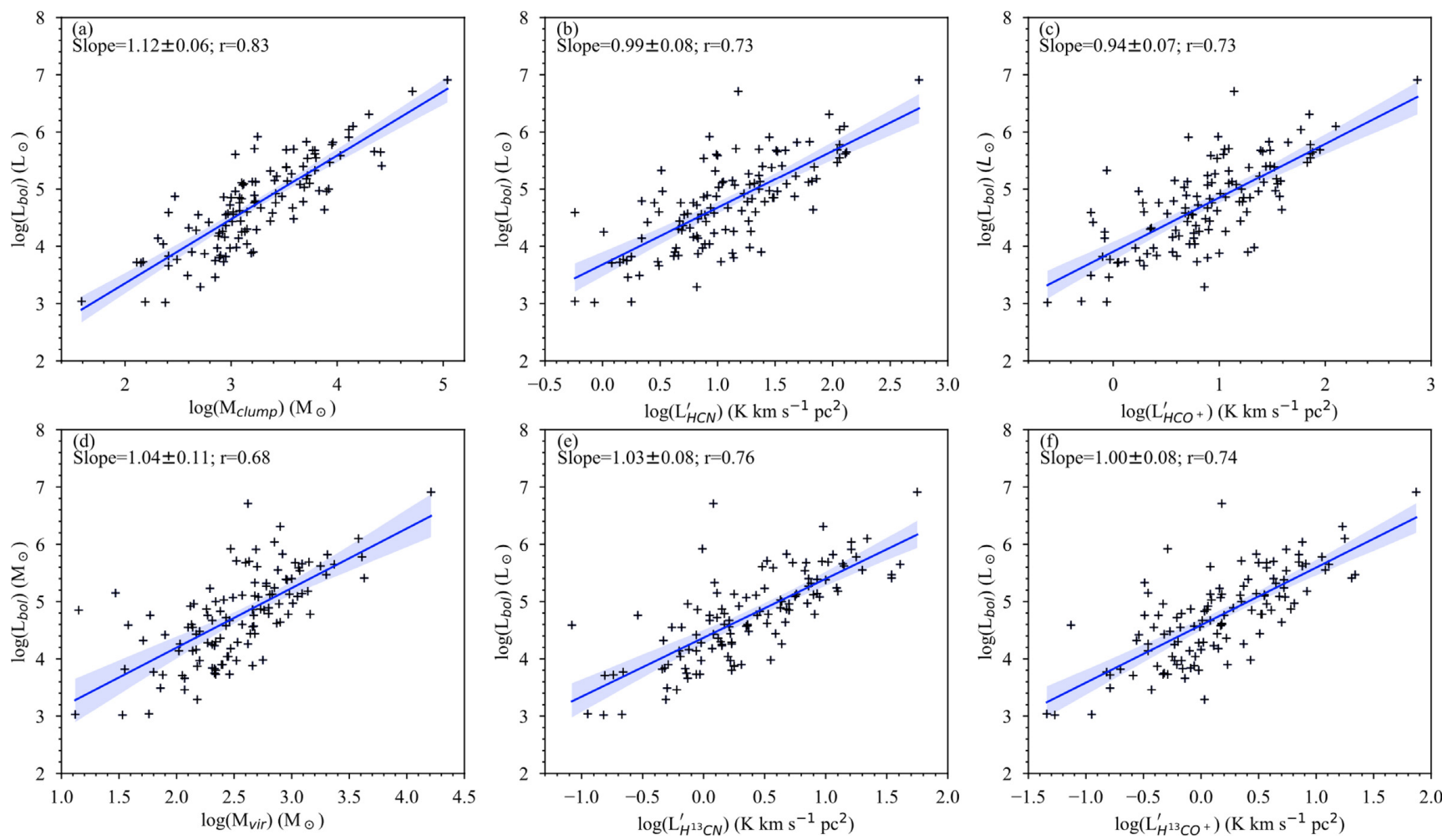

Figure 5. The correlations between $L_{\mathrm{bol}}$ and other parameters $\left(M_{\mathrm{clump}}, M_{\mathrm{vir}}\right.$ derived from $\mathrm{H}^{13} \mathrm{CO}^{+}$and $\left.L_{\text {mol }}^{\prime}\right)$. The solid lines are linear fits. The shadow regions represent 95 per cent confidence levels of the fits. (a) $L_{\mathrm{bol}}$ versus $M_{\mathrm{clump}}$; (b) $L_{\mathrm{bol}}$ versus $L_{\mathrm{HCN}}^{\prime}$; (c) $L_{\mathrm{bol}}$ versus $L_{\mathrm{HCO}}^{\prime}$; (d) $L_{\mathrm{bol}}$ versus $M_{\mathrm{vir}}$ derived from $\mathrm{H}^{13} \mathrm{CO}^{+}$; (e) $L_{\mathrm{bol}}$ versus $L_{\mathrm{H}^{13} \mathrm{CN}}^{\prime}$; (c) $L_{\mathrm{bol}}$ versus $L_{\mathrm{H}^{13} \mathrm{CO}^{+}}^{\prime}$.

tests in Table 3 indicate that they can be drawn from a same distribution. It implies that the optical depths of the main lines do not affect the determination of $\mathrm{HCN}$-to- $\mathrm{HCO}^{+}$ratios statistically. The mean $\mathrm{HCN}$-to- $\mathrm{HCO}^{+}$ratios inferred from distributions of $\log \left(\frac{\mathrm{HCN}}{\mathrm{HCO}^{+}}\right)$and $\log \left(\frac{\mathrm{H}^{13} \mathrm{CN}}{\mathrm{H}^{13} \mathrm{CO}^{+}}\right)$are $\sim 1.5$ and $\sim 1.6$, respectively. This mean $\mathrm{HCN}$-to- $\mathrm{HCO}^{+}$ratio $(1.5-1.6)$ is slightly larger than that in nearby disc galaxies (1.3-1.4; Jiménez-Donaire et al. 2019), Orion B GMC (0.9-1.1; Pety et al. 2017; Shimajiri et al. 2017) and Galactic
Plane GMCs (1-1.4; Nguyen-Luong et al. 2020; Wang et al. 2020). The mean $\mathrm{HCN}-$ to- $\mathrm{HCO}^{+}$ratio in the 'ATOMS' sample is similar to that of Aquila GMC (1.6; Shimajiri et al. 2017). The line ratio of $\mathrm{HCN}-$ to- $\mathrm{HCO}^{+}$seems to be sensitive to environments (Pety et al. 2017; Shimajiri et al. 2017). High $\mathrm{HCN}$-to- $\mathrm{HCO}^{+}$ratios have been found in far-UV irradiated environments such as evolved Galactic H II regions (Nguyen-Luong et al. 2020), AGNs (Aladro et al. 2015), or luminous infrared galaxies (LIRGs; Papadopoulos 2007). This 
Table 2. Correlations between parameters.

\begin{tabular}{lccc}
\hline Relation & $a$ & $b$ & $r$ \\
\hline$M_{\text {clump }}-L_{\text {bol }}$ & $1.12(0.06)$ & $1.12(0.21)$ & 0.83 \\
$M_{\text {vir }}-L_{\text {bol }}$ & $1.04(0.11)$ & $2.12(0.31)$ & 0.68 \\
$M_{\text {vir }}-M_{\text {clump }}$ & $0.83(0.08)$ & $1.14(0.22)$ & 0.73 \\
$L_{\mathrm{HCN}}^{\prime}-L_{\text {bol }}$ & $0.99(0.08)$ & $3.68(0.11)$ & 0.73 \\
$L_{\mathrm{HCO}}^{\prime}-L_{\text {bol }}$ & $0.94(0.07)$ & $3.91(0.09)$ & 0.73 \\
$L_{\mathrm{H}^{13} \mathrm{CN}^{\prime}}^{\prime} L_{\text {bol }}$ & $1.03(0.08)$ & $4.37(0.07)$ & 0.76 \\
$L_{\mathrm{H}^{13} \mathrm{CO}^{+}}-L_{\text {bol }}$ & $1.00(0.08)$ & $4.59(0.05)$ & 0.74 \\
\hline
\end{tabular}

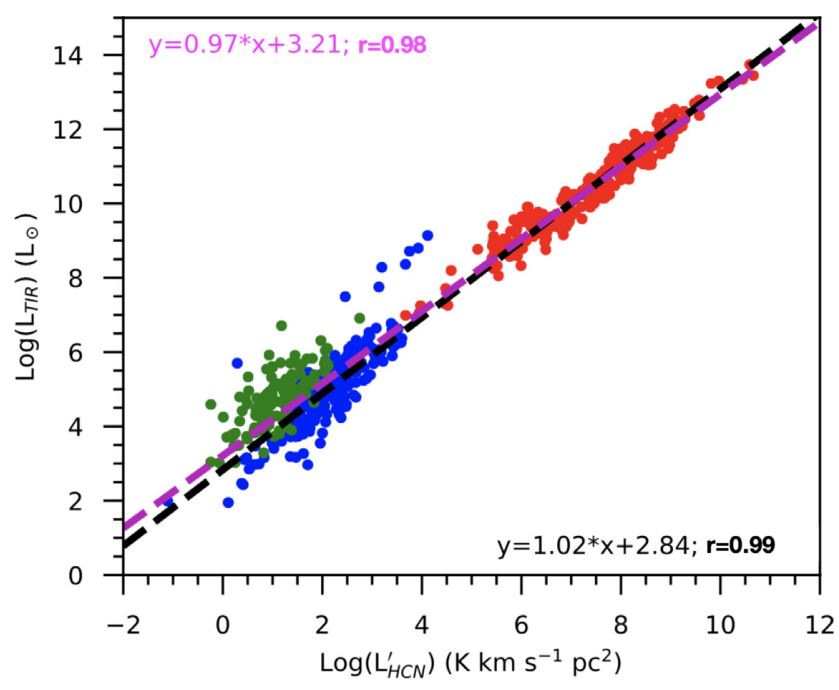

Figure 6. The correlation between $L_{\mathrm{TIR}}$ and $L_{\mathrm{HCN}}^{\prime}$. The data for compact objects in this study are shown as the green filled circles. The data points for Galactic clumps (the blue filled circles) and external galaxies (the red filled circles) in single-dish observations were compiled by Jiménez-Donaire et al. (2019). The linear fit of all data is shown as the the magenta-dashed line. The linear fit towards single-dish data is shown as the black-dashed line.

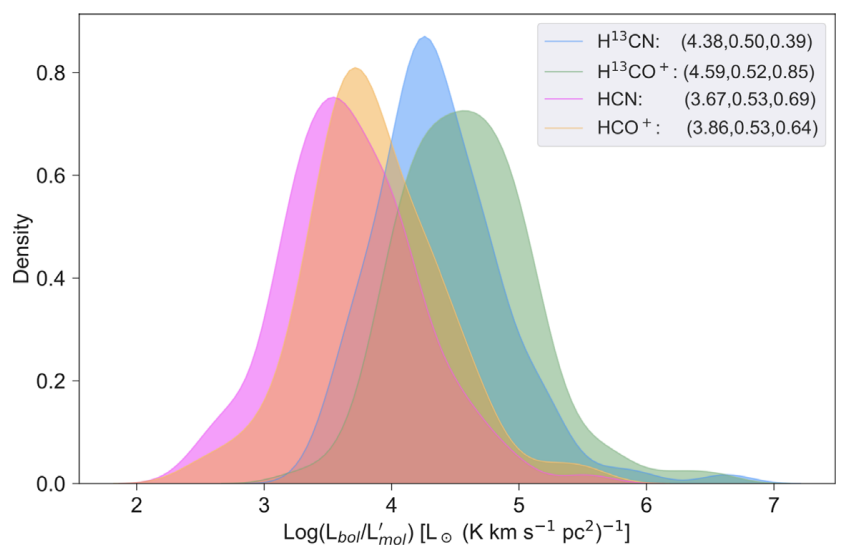

Figure 7. Density distributions of $L_{\mathrm{bol}}$-to- $L_{\mathrm{mol}}^{\prime}$ ratios, plotted using the function 'kdeplot' in the PYTHON package SEABORN. The numbers in brackets are mean, standard deviation, and asymptotic significance in Kolmogorov-Sminov test of a normal distribution. implies that $\mathrm{HCO}^{+}$abundance is sensitive to the ionization degree of molecular gas. In more far-UV irradiated environments, $\mathrm{HCO}^{+}$ is more easily recombined with free electrons, leading to a decrease in its abundance. Increasing $\mathrm{HCN}$-to- $\mathrm{HCO}^{+}$ratios with increasing infrared luminosity have been found in LIRGs (Papadopoulos 2007). In the Galaxy, more evolved H II regions also show higher $\mathrm{HCN}$-to- $\mathrm{HCO}^{+}$ratios than infrared dark clouds (Nguyen-Luong et al. 2020). However, in our data, we do not see any trend of HCNto- $\mathrm{HCO}^{+}$ratios against the $L_{\mathrm{bol}}$ spanning 4 orders of magnitude. This may imply that the majority of $\mathrm{HCN}$ and $\mathrm{HCO}^{+} J=1-0$ line emission within the 'ATOMS' clumps comes from regions that are not exposed to strong local FUV radiation field. This scenario needs to be tested from detailed analysis of the spatial distributions of molecular line emission inside the clumps, which will be presented in forthcoming works using the high resolution ( $2 \operatorname{arcsec})$ ALMA 12-m array data of the 'ATOMS' survey (see Liu et al. 2020, for example).

The density distributions for $\log \left(\frac{\mathrm{HCN}}{\mathrm{H}^{13} \mathrm{CN}}\right)$ and $\log \left(\frac{\mathrm{HCO}^{+}}{\mathrm{H}^{13} \mathrm{CO}^{+}}\right)$are also quite similar to each other but with large scatter $(\sigma \sim 0.6)$ in the data (see Fig. 12). The large scatter means that the optical depths of the main lines show significant variations among sources. The non-parametric tests in Table 3 confirm that their distributions are nearly the same after shifting the distributions by their mean values, indicating that $\mathrm{HCN} J=1-0$ and $\mathrm{HCO}^{+} J=1-0$ may have similar opacity in sources of the 'ATOMS' sample. The mean $\mathrm{HCN}$-to- $\mathrm{H}^{13} \mathrm{CN}$ ratio and $\mathrm{HCO}^{+}$-to- $\mathrm{H}^{13} \mathrm{CO}^{+}$ratio are $\sim 5.0$ and $\sim 5.4$, respectively. Assuming an isotopic ${ }^{12} \mathrm{C} /{ }^{13} \mathrm{C}$ ratio of 55 at the median galactocentric distance of $5.8 \mathrm{kpc}$ (Milam et al. 2005), the representative optical depths inferred from these mean ratios are $\sim 12$ and $\sim 11$ for $\mathrm{HCN} J=1-0$ and $\mathrm{HCO}^{+} J=1-0$, respectively. Thus, the column densities of $\mathrm{HCN}$ and $\mathrm{HCO}^{+}$derived from the main lines with optically thin assumption could be underestimated by a factor of $\sim 10$ for such high optical depths.

However, there is no trend between molecular line luminosity ratios ( $\mathrm{HCN}$-to- $\mathrm{HCO}^{+}, \mathrm{H}^{13} \mathrm{CN}$-to- $\mathrm{H}^{13} \mathrm{CO}^{+}, \mathrm{HCN}$-to- $\mathrm{H}^{13} \mathrm{CN}$ ratio, and $\mathrm{HCO}^{+}$-to- $\mathrm{H}^{13} \mathrm{CO}^{+}$) and $L_{\mathrm{bol}}$ [see panels (e)-(h) in Fig. 8] All these molecular line luminosity ratios seem constant against $L_{\text {bol }}$ spanning 4 orders of magnitude in $L_{\text {bol }}$. This implies that optical depths (or abundance ratios) of main lines should not affect the interpretation of the slopes in star formation relations. The molecular line luminosity ratios are also not correlated with dust temperature [see panels (e)-(h) in Fig. 9] or clump masses [see panels (e)-(h) in Fig. 10].

Panels (e)-(h) in Fig. 11 present correlations between molecular line luminosity ratios and galactocentric distances $\left(R_{\mathrm{GC}}\right)$ for different tracers. In a survey of nine nearby external galaxies, JiménezDonaire et al. (2019) found that there is a decreasing trend of $\mathrm{HCN}-$ to- $\mathrm{HCO}^{+}$as a function of galactocentric distances in only two galaxies. No significant changing $\mathrm{HCN}-$ to- $-\mathrm{HCO}^{+}$ratios are witnessed in the other seven galaxies. There is also no obvious trend for molecular line luminosity ratios against $R_{\mathrm{GC}}$ in our data, indicating similar local environments (such as FUV radiation field, density, temperature) for the sources in the 'ATOMS' sample.

\subsection{Star formation efficiencies}

Until now, we have dealt with observables, comparing luminosities and masses of various quantities. Now, we engage in the more speculative issues of SFR and SFE. We use the extragalactic definition of SFE: the SFR per unit mass of gas, with units of $\mathrm{Myr}^{-1}$. On the scale of galaxies and averaged over $5 \mathrm{Myr}$ or longer, the far-infrared luminosity can measure the SFR. The relation given 

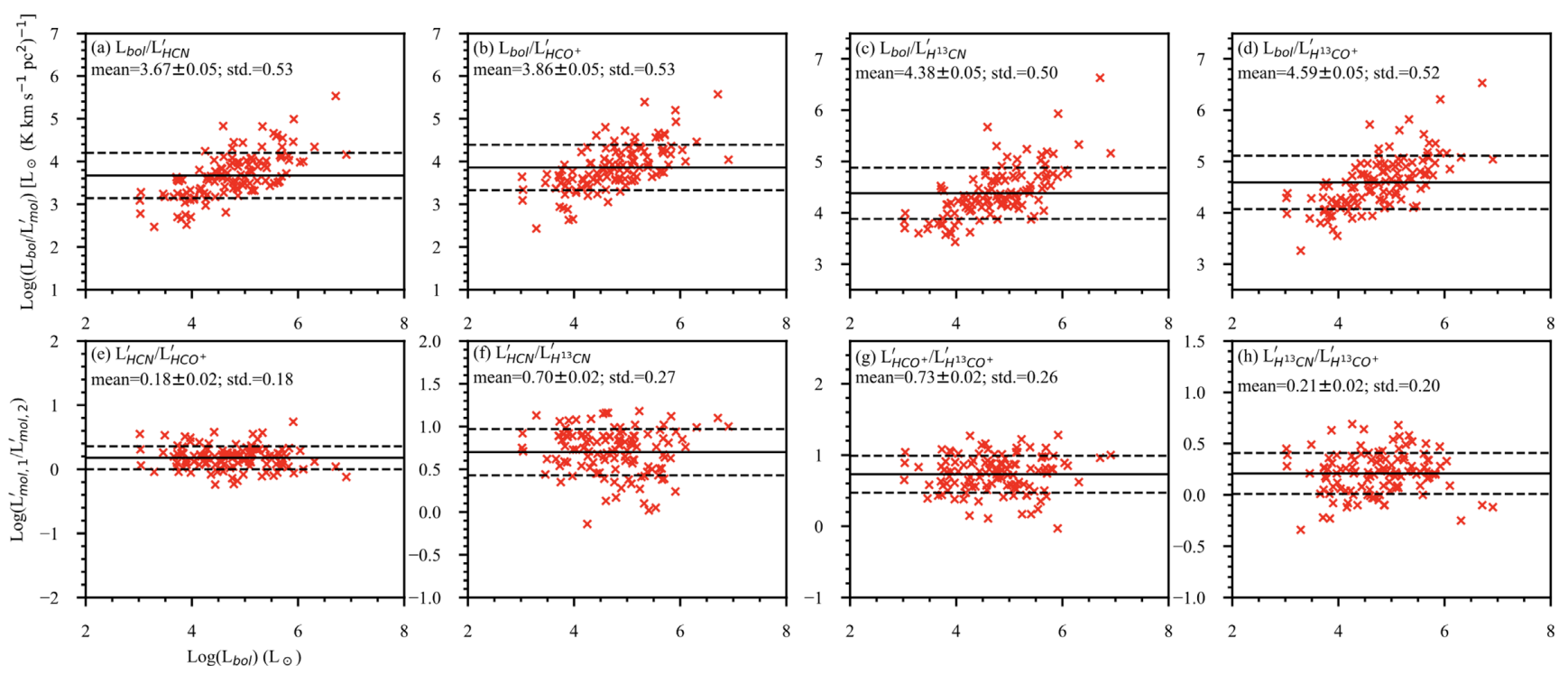

Figure 8. $L_{\mathrm{bol}}$-to- $L_{\mathrm{mol}}^{\prime}$ ratios (panels a-d) and line luminosity ratios (panels e-h) as a function of bolometric luminosity $L_{\mathrm{bol}}$. The solid line is the mean value. The dashed lines show standard deviation. The name of the ratio is labelled at the upper right corner in each panel.

Table 3. Asymptotic significances (P-value) in non-parametric tests.

\begin{tabular}{lc}
\hline Distributions & Kolmogorov-Sminov $^{a}$ \\
\hline $\log \left(\frac{L_{\text {bol }}}{\mathrm{H}^{13} \mathrm{CN}}\right)$ versus $\log \left(\frac{L_{\text {bol }}}{\mathrm{H}^{13} \mathrm{CO}}\right)$ & 0.69 \\
$\log \left(\frac{L_{\text {bol }}}{\mathrm{HCN}}\right)$ versus $\log \left(\frac{L_{\text {bol }}}{\mathrm{HCO}}\right)$ & 0.97 \\
$\log \left(\frac{L_{\text {bol }}}{\mathrm{H}^{13} \mathrm{CN}}\right)$ versus $\log \left(\frac{L_{\text {blol }}}{\mathrm{HCN}}\right)$ & 0.95 \\
$\log \left(\frac{L_{\text {bol }}}{\mathrm{H}^{13} \mathrm{CO}}\right)$ versus $\log \left(\frac{L_{\text {bol }}}{\mathrm{HCO}^{+}}\right)$ & 0.95 \\
$\log \left(\frac{\mathrm{HCN}}{\mathrm{HCO}^{+}}\right)$versus $\log \left(\frac{\mathrm{H}^{13} \mathrm{CN}^{13}}{\mathrm{H}^{13} \mathrm{CO}^{+}}\right)$ & 0.89 \\
$\log \left(\frac{\mathrm{HCN}}{\mathrm{H}^{13} \mathrm{CN}}\right)$ versus $\log \left(\frac{\mathrm{HCO}^{+}}{\mathrm{H}^{13} \mathrm{CO}^{+}}\right)$ & 0.89
\end{tabular}

${ }^{a}$ The significance level ( $\alpha$-value) is 0.05 .

in table 1 of Kennicutt \& Evans (2012), and explained in more detail in Hao et al. (2011) and Murphy et al. (2011), can be written in convenient units as

$\mathrm{SFR}=1.49 \times 10^{-4} L_{\mathrm{bol}}\left(\mathrm{L}_{\odot}\right) \mathrm{M}_{\odot} \mathrm{Myr}^{-1}$.

This conversion depends on assumptions about initial mass function (IMF) and star formation history, so there is no guarantee that it would apply to the spatial and temporal scales studied in this paper. However, we wish to compare the current results to those of other works that used different indicators of SFR. Consequently, we use the equation above to generate estimates of SFR and compare to various mass estimates to gauge the SFE. Comparison of SFE, as opposed to SFR, among samples is best suited to appreciate the dispersion and the relative quality of different predictors of star formation, as shown by Vutisalchavakul et al. (2016); so we focus on SFE here. We further follow Vutisalchavakul et al. (2016) in computing means, medians, and standard deviations of SFE in the $\log$, and present them in Table 4.

As predictors, we consider three estimates of the dense gas mass. These estimates also come with various caveats about what characteristic density they represent (Evans et al. 2020). The first estimate is the masses ( $M_{\text {clump }}$ in Table A6) of clumps from single dish observations, which were obtained from fitting SEDs using farinfrared (IRAS or Herschel) data and (sub-)millimeter continuum data $(0.87 \mathrm{~mm}$ from Urquhart et al. 2018 or $1.2 \mathrm{~mm}$ from Faúndez et al. 2004). We also use, secondly, the virial masses derived from both $\mathrm{HCO}^{+}$and $\mathrm{H}^{13} \mathrm{CO}^{+}\left(M_{\text {vir }}^{12}\right.$ and $M_{\text {vir }}^{13}$ in Table A6).

We compare our results for SFE to those of Vutisalchavakul et al. (2016) first. They determined the SFR from the mid-infrared luminosity, again using the extra-galactic relations, which carry the same caveat about IMF and star formation history discussed above. However, they did find that the SFR from the mid-infrared luminosity agreed well with those from the free-free radio continuum, which averages over times of 3-10 Myr, as long as the inferred SFR was at least $5 \mathrm{M}_{\odot} \mathrm{Myr}^{-1}$. The criterion of $\log L_{\mathrm{bol}} \geq 4.5$ used by Wu et al. (2010) implies a SFR of $4.7 \mathrm{M}_{\odot} \mathrm{Myr}^{-1}$, essentially the same criterion. Of the 119 sources in Table A6, 75 satisfy this criterion. Also, the sample of Vutisalchavakul et al. (2016) was defined by submillimeter continuum emission, which traced relatively large, somewhat dense clumps. If we make the cut between cores and clumps at a size of $0.2 \mathrm{pc}$ (based on $R_{\text {eff }}$ in table 6), all 75 sources that made the first cut also satisfy the size criteria that make them directly comparable to the sample of Vutisalchavakul et al. (2016).

Fig. 13 plots the $\log$ of SFE versus the $\log$ of $M_{\text {clump }}$ for sources that do (black) and do not (red) meet the requirement on SFR of $5 \mathrm{M}_{\odot} \mathrm{Myr}^{-1}$. There are no obvious overall trends in SFE within each sample, but the low luminosity sources lie clearly below the ones above the threshold previously used (Wu et al. 2010; Vutisalchavakul et al. 2016). There are five clear outliers (I09002-4732, I12320-6122, I16562-3959, I17258-3637, and I18139-1842) in Fig. 13 at high SFE. These turn out to have unusually large values for $T_{\mathrm{d}}(\gtrsim 39 \mathrm{~K})$, as listed in Table A6. To the extent that $T_{\mathrm{d}}$ reflects evolutionary state, these sources would be more evolved. If so, a higher fraction of their masses would have been converted into stars, leading to a higher SFE. Table 4 shows the logarithmic means, medians, and standard deviations of the full sample, along with those with the restrictions applied, for three estimates of the mass of dense gas. While the cuts decrease the means and medians, the differences are not large (about $0.3 \mathrm{dex}$ ). The last line in Table 4 shows the statistics for the 44 sources that lie below the cut in SFR of $5 \mathrm{M}_{\odot} \mathrm{Myr}^{-1}$; their mean and median SFE are much lower, reflecting the fact that $\log L_{\text {bol }}$ no longer traces the SFR below that value. The SFE based on the $\mathrm{HCO}^{+}$virial mass and the single-dish 

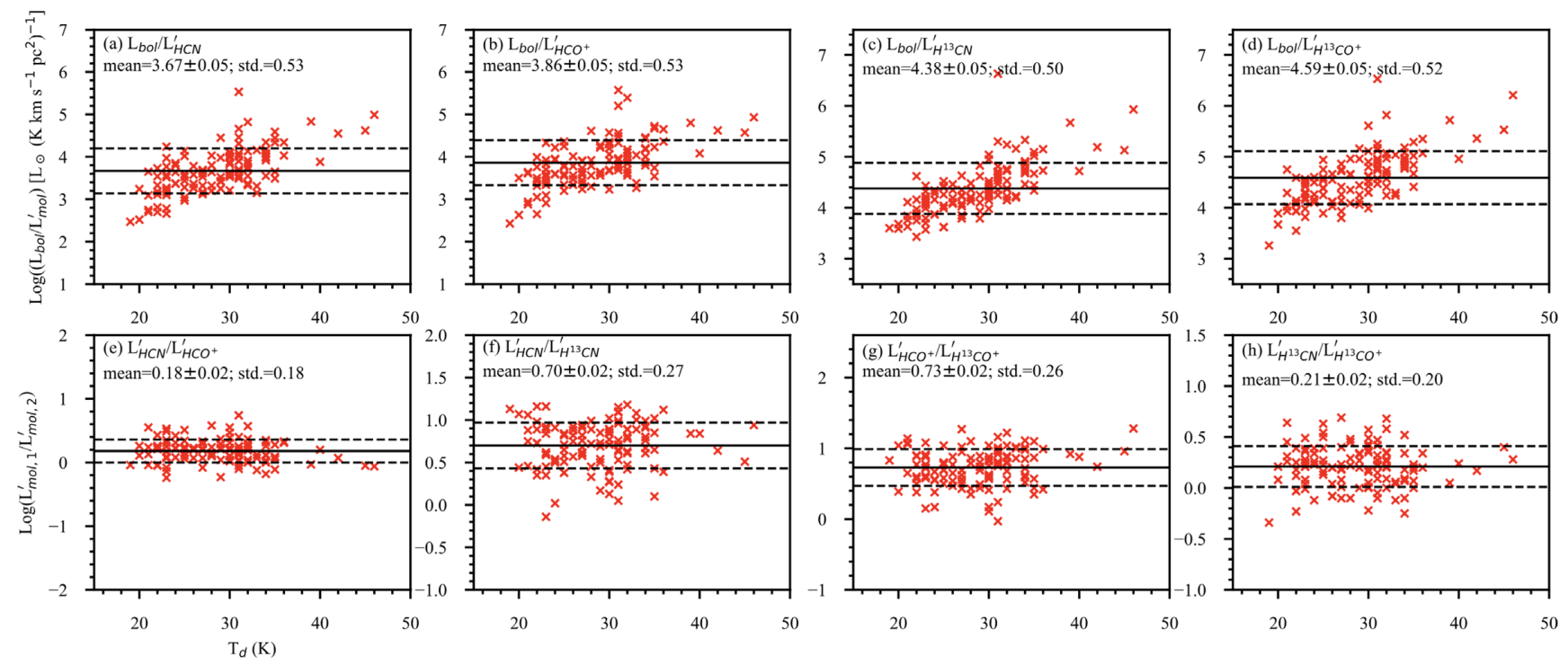

Figure 9. $L_{\mathrm{bol}}$-to- $L_{\text {mol }}^{\prime}$ ratios (panels a-d) and line luminosity ratios (panels e-h) as a function of dust temperature $T_{\mathrm{d}}$. The solid line is the mean value. The dashed lines show standard deviation. The name of the ratio is labelled at the upper right corner in each panel.
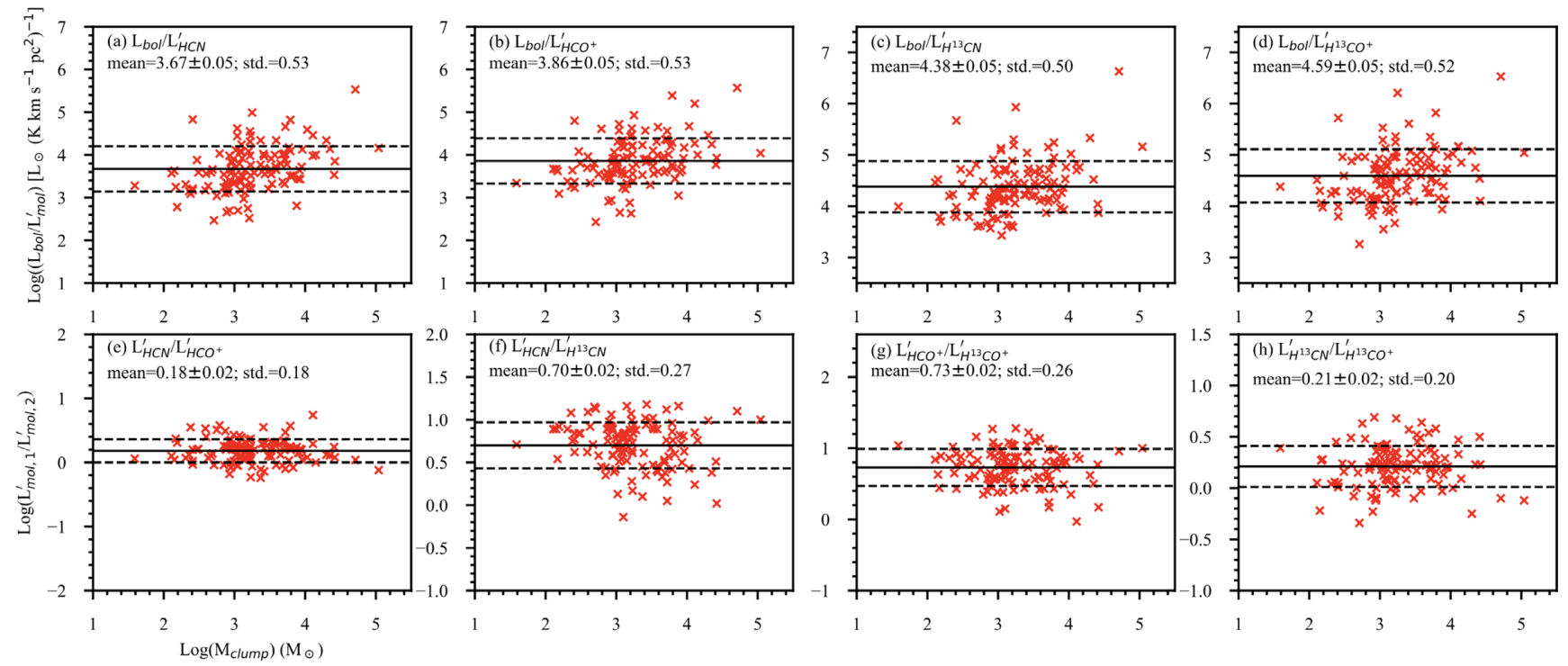

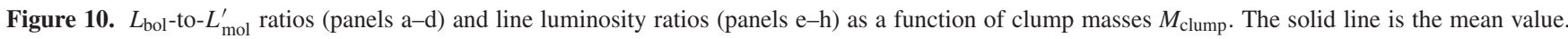
The dashed lines show standard deviation. The name of the ratio is labelled at the upper right corner in each panel.

clump mass are quite similar, but the latter has a smaller dispersion (0.36 dex). The SFE based on the virial mass from $\mathrm{H}^{13} \mathrm{CO}^{+}$is larger by about 0.8 dex. The mean value for the log of SFE from Vutisalchavakul et al. (2016) is $-1.74 \pm 0.50$ for the dense gas, with mean volume density of $n \sim 10^{3} \mathrm{~cm}^{-3}, 0.38$ dex higher than the current sample. In turn, the sample of nearby clouds, where the dense gas mass was determined from the condition that $A_{V} \geq 8 \mathrm{mag}$, had a still higher SFE, about -1.61 . The SFR in the nearby clouds was measured by young stellar object (YSO) counting, which is much less sensitive to the IMF and star formation history (Evans, Heiderman \& Vutisalchavakul 2014).

The masses of dense gas for this sample $\left(\left\langle\log \left(M_{\text {clump }}\right)\right\rangle=3.24\right.$; see Table 1) seem to be similar to that of Vutisalchavakul et al. (2016; $\left.\left\langle\log \left(M_{\text {clump }}\right)\right\rangle \sim 3.4\right)$. However, the sizes of the regions are somewhat smaller with a mean effective clump radius of $1.1 \mathrm{pc}$ (see
Table 1), versus $3.1 \pm 2.1 \mathrm{pc}$ for the sample of Vutisalchavakul et al. (2016) . Consequently, the surface and volume densities are higher. The sample selection may play a role. This sample was selected from a survey of CS $J=2-1$ emission (though that survey was itself directed towards IRAS point sources with infrared colours similar to UC H II regions), thus focusing on relatively dense gas, while the sample of Vutisalchavakul et al. (2016) began with surveys of radio recombination lines, requiring recent formation of massive stars. Based on smaller sizes and higher surface densities, this sample may be younger than the sample of Vutisalchavakul et al. (2016), so a smaller SFE would be expected.

Despite the differences, the values from this paper lie well within the dispersion of values from Vutisalchavakul et al. (2016) and are consistent with the nearly constant SFE from nearby clouds to distant galaxies shown in fig. 12 of Vutisalchavakul et al. (2016). We 

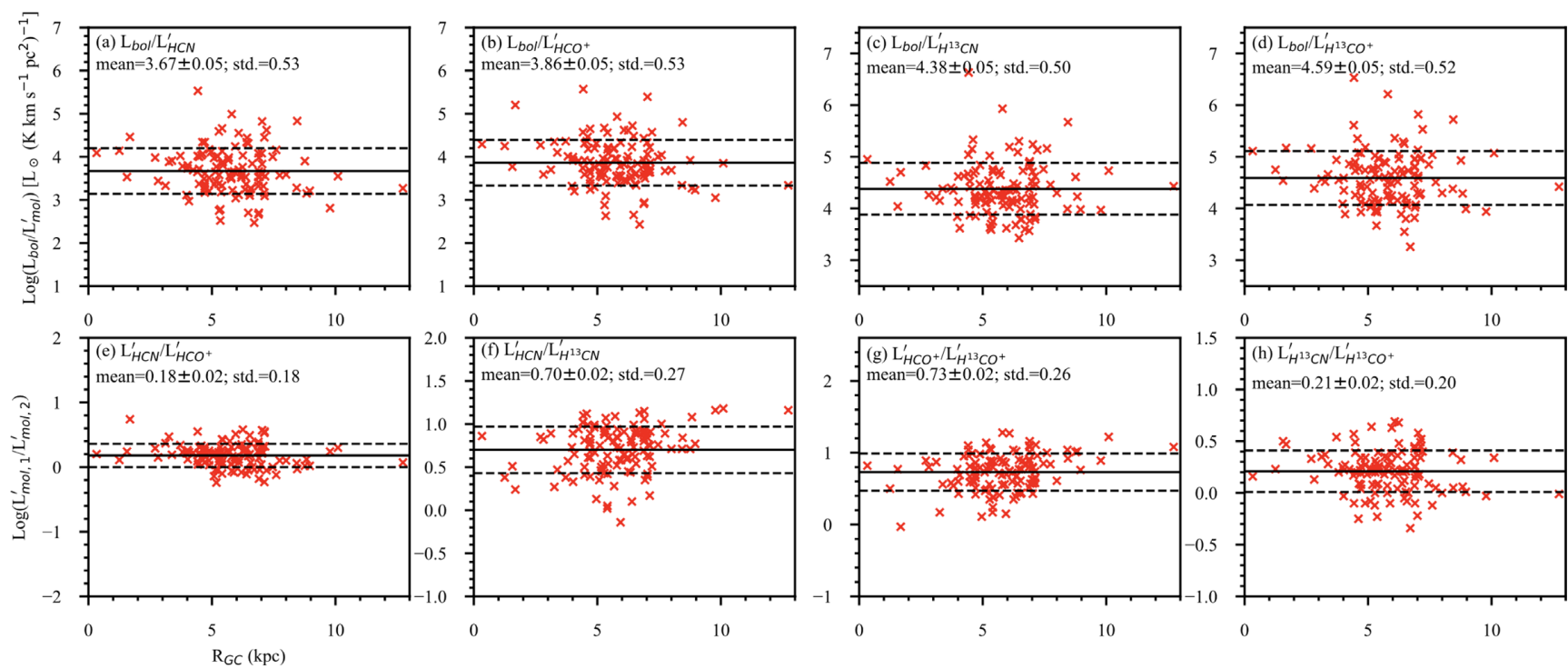

Figure 11. $L_{\mathrm{bol}}$-to- $L_{\mathrm{mol}}^{\prime}$ ratios (panels a-d) and line luminosity ratios (panels e-h) as a function of galactocentric distances. The name of the ratio is labelled at the upper right corner in each panel.

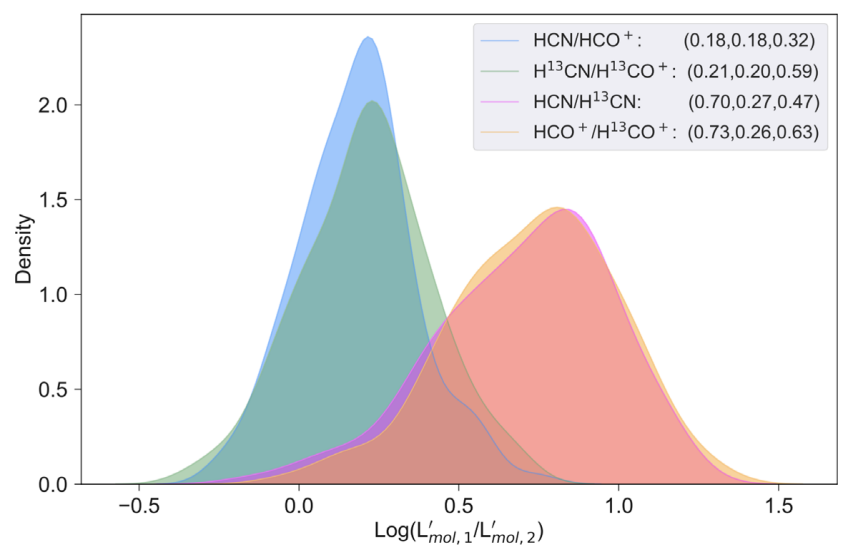

Figure 12. Density distributions of molecular line luminosity ratios, plotted using the function 'kdeplot' in the PYTHON package SEABORN. The numbers in brackets are mean, standard deviation, and asymptotic significance in Kolmogorov-Sminov test of a normal distribution.

reproduce that figure here (see Fig. 14), with the points for ATOMS and for the EMPIRE study (Jiménez-Donaire et al. 2019) added.

\section{CONCLUSIONS}

Some molecular gas tracers (e.g. $\mathrm{HCN}$ and $\mathrm{HCO}^{+}$) that are commonly used in studies of external galaxies have large optical depths and may not be good tracers of the distribution of dense molecular gas in molecular clouds. In this work, we use the isotopologues of $\mathrm{HCN}$ and $\mathrm{HCO}^{+}$to calibrate the relationships between the recent SFR, as traced by the total infrared emission, and the dense molecular gas mass determined from line luminosities $\left(L_{\text {mol }}^{\prime}\right)$. The data used in this work are from ACA observations in the 'ATOMS' survey of 146 active Galactic star-forming regions. Our main results are summarized as follows:

(1) We extracted 173,184, 190, 189, and 182 compact objects from $3 \mathrm{~mm}$ continuum emission, $\mathrm{H}^{13} \mathrm{CN} J=1-0, \mathrm{H}^{13} \mathrm{CO}^{+} J=$ $1-0, \mathrm{HCN} J=1-0$, and $\mathrm{HCO}^{+} J=1-0$ line emission, respectively. The compact objects in $3 \mathrm{~mm}$ continuum emission are systematically smaller than compact objects in molecular line emission. Compact sources in $\mathrm{HCN} J=1-0$, and $\mathrm{HCO}^{+}$ $J=1-0$ line emission have statistically larger effective radii than the compact objects found in the line emission of their isotopologues.

(2) The virial masses of compact objects in $\mathrm{H}^{13} \mathrm{CO}^{+} J=1-0$ line emission are systematically smaller than the total masses of their clumps with a median virial parameter $\left(\alpha=M_{\text {vir }} / M_{\text {clump }}\right)$ of $\sim 0.2$, While the virial masses from $\mathrm{HCO}^{+}$are comparable to the total masses of their natal clumps with a median virial parameter of $\sim 1.2$. It suggests that our ACA observations most likely detect the gravitationally bound structures inside clumps.

(3) All the correlations between $L_{\mathrm{bol}}$, tracing the SFR, and molecular line luminosities $L_{\text {mol }}^{\prime}$ of the four transitions $\left(\mathrm{H}^{13} \mathrm{CN}\right.$ $J=1-0, \mathrm{H}^{13} \mathrm{CO}^{+} J=1-0, \mathrm{HCN} J=1-0$, and $\mathrm{HCO}^{+} J=$ $1-0)$ appear approximately linear. Line emission of isotopologues shows scatter in their $L_{\mathrm{bol}}-L_{\text {mol }}^{\prime}$ relations as large as that found in their main line emission. Although the main lines may not be good tracers of the spatial distribution of dense gas in molecular clouds (Pety et al. 2017; Shimajiri et al. 2017), they trace the total dense gas masses as well as do their isotopologues.

(4) The density distributions of $\log \left(L_{\mathrm{bol}} / L_{\mathrm{mol}}^{\prime}\right)$ for different molecular line tracers show high similarity, indicating that they can be drawn from a same underlying distribution, again confirming that the main lines trace the total dense gas masses as well as do their isotopologues. There seems to be a clear trend between increasing $L_{\mathrm{bol}}$-to- $L_{\text {mol }}^{\prime}$ ratios and $L_{\mathrm{bol}}$ (also $T_{\mathrm{d}}$ ) spanning four orders of magnitude in $L_{\mathrm{bol}}$. This is likely caused by evolutionary effects in the sources. $L_{\mathrm{bol}}$-to- $L_{\text {mol }}^{\prime}$ ratios do not vary with galactocentric distance $\left(R_{\mathrm{GC}}\right)$, suggesting that SFEs remain constant for gravitationally bound clumps in different Galactic environments.

(5) All the molecular line luminosity ratios $\left(\mathrm{HCN}-\right.$ to- $-\mathrm{HCO}^{+}$, $\mathrm{H}^{13} \mathrm{CN}$-to- $\mathrm{H}^{13} \mathrm{CO}^{+}, \mathrm{HCN}$-to- $\mathrm{H}^{13} \mathrm{CN}$ ratio and $\mathrm{HCO}^{+}$-to- $\mathrm{H}^{13} \mathrm{CO}^{+}$) appear constant when compared to $L_{\mathrm{bol}}$ over four orders of magnitude in $L_{\text {bol }}$. This implies that the large optical depths (or abundance ratios) of main lines do not affect the interpretation of the slopes in star formation relations. There is also no obvious overall trend for 
Table 4. Statistics of the log of star formation efficiency.

\begin{tabular}{|c|c|c|c|c|c|c|c|c|c|c|}
\hline \multirow[b]{2}{*}{ Number } & \multicolumn{3}{|c|}{$\mathrm{SFR} / \mathrm{M}_{\mathrm{vir}}\left(\mathrm{HCO}^{+}\right)$} & \multicolumn{3}{|c|}{$\mathrm{SFR} / \mathrm{M}_{\text {clump }}$} & \multicolumn{3}{|c|}{$\mathrm{SFR} / \mathrm{M}_{\mathrm{vir}}\left(\mathrm{H}^{13} \mathrm{CO}^{+}\right)$} & \multirow[b]{2}{*}{ Selection $^{a}$} \\
\hline & Mean & Median & Std & Mean & Median & Std & Mean & Median & Std & \\
\hline 119 & -2.38 & -2.33 & 0.63 & -2.33 & -2.29 & 0.43 & -1.62 & -1.66 & 0.55 & All \\
\hline 75 & -2.09 & -2.11 & 0.50 & -2.13 & -2.10 & 0.36 & -1.35 & -1.39 & 0.46 & $\mathrm{SFR}>5$ \\
\hline 75 & -2.09 & -2.11 & 0.50 & -2.13 & -2.10 & 0.36 & -1.35 & -1.39 & 0.46 & $\mathrm{SFR}>5, r>0.2$ \\
\hline 44 & -2.89 & -2.79 & 0.50 & -2.66 & -2.69 & 0.32 & -2.08 & -2.13 & 0.35 & $\mathrm{SFR}<5, r>0.2$ \\
\hline
\end{tabular}

${ }^{a}$ Selection criteria explained in the text.

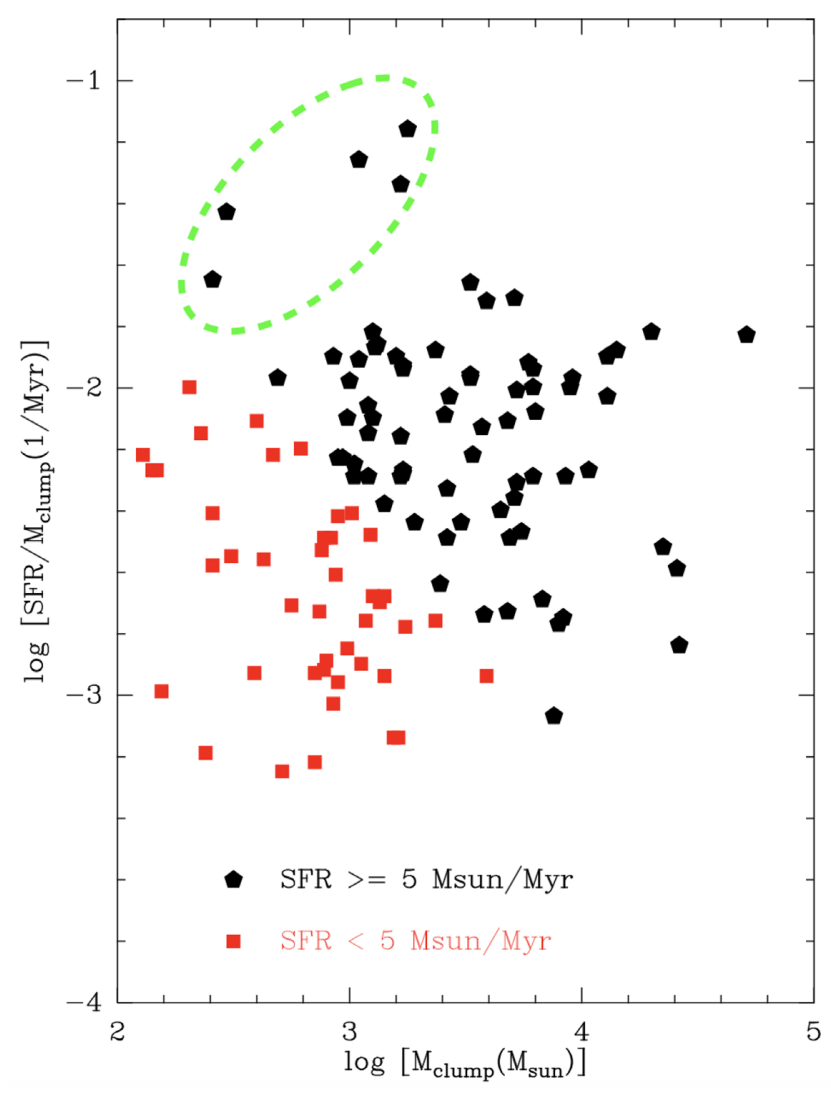

Figure 13. SFR $/ M_{\text {clump }}$ is plotted versus $M_{\text {clump }}$. The red points represent the sources with SFR $<5 \mathrm{M}_{\odot} \mathrm{Myr}^{-1}$, while the black points represent the sources with $\mathrm{SFR} \geq 5 \mathrm{M}_{\odot} \mathrm{Myr}^{-1}$. The points inside the green-dashed ellipse are five outliers with highest dust temperature in the sample.

molecular line luminosity ratios against $T_{\mathrm{d}}, M_{\text {clump }}$, and $R_{\mathrm{GC}}$ in our data.

(6) If we use extragalactic calibrations to convert $L_{\text {bol }}$ to a SFR and compare it to masses of clumps from single-dish observations or to virial masses from $\mathrm{HCO}^{+}$, we can estimate a dense gas SFE. While this procedure is not fully justified for the scales we are studying, we find that the logarithmic mean SFE is reasonably consistent with other measures of SFE for dense gas, even those using very different tracers or examining very different spatial scales.

\section{ACKNOWLEDGEMENTS}

TL is supported by the initial funding of scientific research for high-level talents at Shanghai Astronomical Observatory. This work was carried out in part at the Jet Propulsion Laboratory, which is operated for NASA by the California Institute of

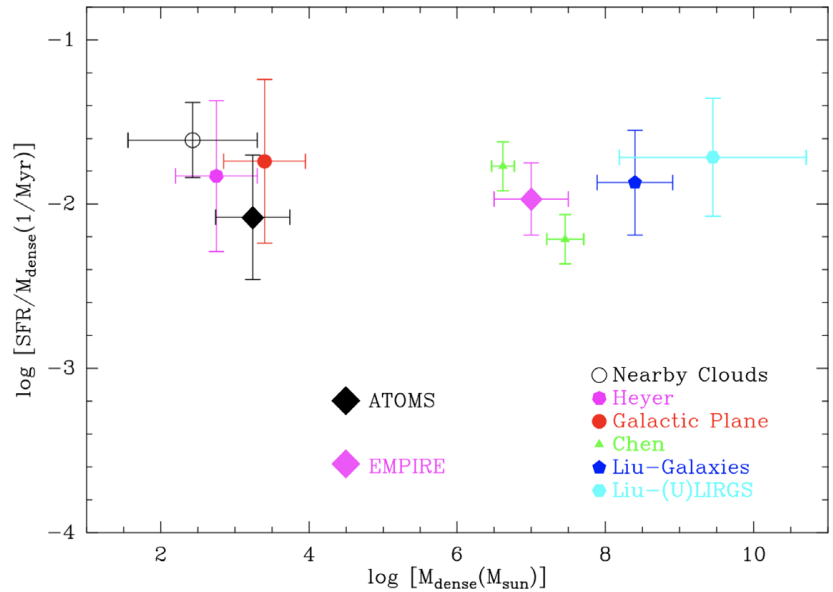

Figure 14. The logarithmic mean SFE (SFR/ $M_{\text {dense }}$ ) is plotted versus $M_{\text {dense }}$ with both showing the standard deviation in the log. The black diamond is from this work, and the magenta diamond is from the EMPIRE study of nearby galaxies (Jiménez-Donaire et al. 2019). The other points are the same as those in Vutisalchavakul et al. (2016). The red filled circle is from Vutisalchavakul et al. (2016) for Galactic Plane clouds, the empty black circle is from Evans et al. (2014) for nearby clouds. The magenta point (heptagon) comes from Heyer et al. (2016) for a sample of molecular clouds containing $24 \mu \mathrm{m}$-based Class I protostars. The two green triangles are from Chen et al. (2015) for resolve giant molecular clouds in M51 (lower one for inner galaxy and higher one for outer galaxy). The blue and cyan points represent the whole normal galaxies and the whole (U)LIRGs, respectively, from Chen et al. (2015).

Technology. GG and LB acknowledge support from CONICYT project Basal AFB-170002. CWL is supported by the Basic Science Research Program through the National Research Foundation of Korea (NRF) funded by the Ministry of Education, Science and Technology (NRF-2019R1A2C1010851). Di Li is supported by the CAS International Partnership Program grant No. 114A11KYSB20160008. This paper has used the following ALMA data: ADS/JAO.ALMA\#2019.1.00685.S. ALMA is a partnership of ESO (representing its member states), NSF (USA) and NINS (Japan), together with NRC (Canada), MOST and ASIAA (Taiwan), and KASI (Republic of Korea), in cooperation with the Republic of Chile. The Joint ALMA Observatory is operated by ESO, AUI/NRAO, and NAOJ.

\section{REFERENCES}

Aladro R. et al., 2015, A\&A, 579, A101

Bertoldi F., McKee C. F., 1992, ApJ, 395, 140

Bronfman L., Nyman L.-A., May J., 1996, A\&AS, 115, 81

Chen H., Gao Y., Braine J., Qiusheng G., 2015, ApJ, 810, 140

Evans N. J., II, 1999, ARA\&A, 37, 311

Evans N. J., Heiderman A., Vutisalchavakul N., 2014, ApJ, 782, 114 
Evans, Neal J., II, Kim K.-T., Wu J.-W., 2020, ApJ, 894, 103

Faúndez S., Bronfman L., Garay G., Chini R., Nyman L. -^̊., May J., 2004, A\&A, 426, 97

Gao Y., Solomon P. M., 2004, ApJ, 606, 271

García-Burillo S., Usero A., Alonso-Herrero A., Graciá-Carpio J., PereiraSantaella M., Colina L., Planesas P., Arribas S., 2012, A\&A, 539, A8

Graciá-Carpio J., García-Burillo S., Planesas P., Colina L., 2006, ApJ, 640, L135

Greve T. R. et al., 2014, ApJ, 794, 142

Hao C.-N., Kennicutt R. C., Johnson B. D., Calzetti D., Dale D. A., Moustakas J., 2011, ApJ, 741, 124

Heiderman A., Evans N. J., II, Allen L. E., Huard T., Heyer M., 2010, ApJ, 723,1019

Heyer M., Gutermuth R., Urquhart J. S., Csengeri T., Wienen M., Leurini S., Menten K., Wyrowski F., 2016, A\&A, 588, A29

Hoq S. et al., 2013, ApJ, 777, 157

Hull C. L. H., Zhang Q., 2019, Frontiers Astron. Space Sci., 6, 3

Jiménez-Donaire, María J., Bigiel F., Leroy A. K., 2019, ApJ, 880, 127

Juneau S., Narayanan D. T., Moustakas J., Shirley Y. L., Bussmann R. S., Kennicutt R. C., Jr., Vanden Bout P. A., 2009, ApJ, 707, 1217

Kauffmann J., Pillai T., Goldsmith P. F., 2013, ApJ, 779, 185

Kauffmann J., Goldsmith P. F., Melnick G., Tolls V., Guzman A., Menten K. M., 2017, A\&A, 605, L5

Kennicutt R. C., Evans N. J., 2012, ARA\&A, 50, 531

Lada C. J., Lombardi M., Alves J. F., 2010, ApJ, 724, 687

Lada C. J., Forbrich J., Lombardi M., Alves J. F., 2012, ApJ, 745, 190

Liu D., Gao Y., Isaak K., Daddi E., Yang C., Lu N., van der Werf P., 2015, ApJ, 810L, 14L

Liu T. et al., 2016, ApJ, 829, 59

Liu T. et al., 2018a, ApJ, 859, 151

Liu T. et al., 2018b, ApJ, 869L, 5L

Liu T. et al., 2020, MNRAS, , 496, 2790

McMullin J. P. et al., 2007, Astronomical Data Analysis Software and Systems, XVI, 127

Milam S. N., Savage C., Brewster M. A., Ziurys L. M., Wyckoff S., 2005, ApJ, 634, 1126

Murphy E. J. et al., 2011, ApJ, 737, 67

Narayanan D., Cox T. J., Shirley Y., Dave R., Hernquist L., Walker C. K., 2008, ApJ, 684, 996

Nguyen-Luong Q. et al., 2020, ApJ, 891, 66

Papadopoulos P. P., 2007, ApJ, 656, 792

Pety J. et al., 2017, A\&A, 599, A98

Pillai T., Kauffmann J., Wyrowski F., Hatchell J., Gibb A. G., Thompson M. A., 2011, A\&A, 530, A118

Sanhueza P. et al., 2012, ApJ, 756, 60
Sanhueza P., Jackson J. M., Zhang Q., 2017, ApJ, 841, 97

Shimajiri Y., André P., Braine J., Garay G., Silva A., Finn S. C., 2017, A\&A, 604, A74

Shirley, Yancy L., 2015, PASP, 127, 299

Soam A., Liu T., Andersson B.-G., 2019, ApJ, 883, 95

Solomon P. M., Downes D., Radford S. J. E., Barrett J. W., 1997, ApJ, 478, 144

Stephens I. W., Jackson J. M., Whitaker J. S., 2016, ApJ, 824, 29

Tan Q. et al., 2018, ApJ, 860, 165

Tang Y., Koch P. M., Peretto N., Novak G., Duarte-Cabral A., Chapman N. L., Hsieh P.-Y., Yen H.-W., 2019, ApJ, 878, 10

Urquhart J. S., König C., Giannetti A., 2018, MNRAS, 473, 1059

Vutisalchavakul N., Evans N. J., II, Heyer M., 2016, ApJ, 831, 73

Wang Y. et al., 2020, A\&A, preprint (arXiv:2003.05384)

Wu J., Evans N. J., II, Gao Y., Solomon P. M., Shirley Y. L., Vanden Bout P. A., 2005, ApJ, 635, L173

Wu J., Evans N. J., II, Shirley Y. L., Knez C., 2010, ApJS, 188, 313

Zhang Q., Wang K., Lu X., Jiménez-Serra I., 2015, ApJ, 804, 141

Zhang Z.-Y., Gao Y., Henkel C., Zhao Y., Wang J., Menten K. M., Güsten R., 2014, ApJ, 784, L31

\section{SUPPORTING INFORMATION}

Supplementary data are available at MNRAS online.

Table A1. Selection of Compact objects identified in $3 \mathrm{~mm}$ continuum emission ${ }^{a}$.

Table A2. Selection of compact objects identified in $\mathrm{H}^{13} \mathrm{CO}^{+} J=$ $1-0^{a}$.

Table A3. Selection of compact objects identified in $\mathrm{H}^{13} \mathrm{CN} J=$ $1-0^{a}$.

Table A4. Selection of compact objects identified in $\mathrm{HCO}^{+} \mathrm{J}=1$ $0^{a}$.

Table A5. Selection of compact objects identified in $\mathrm{HCN} J=$ $1-0^{a}$

Table A6. Parameters of selection from 119 sources $^{a}$.

Please note: Oxford University Press is not responsible for the content or functionality of any supporting materials supplied by the authors. Any queries (other than missing material) should be directed to the corresponding author for the article.

\section{APPENDIX A:}

Table A1. Selection of Compact objects identified in $3 \mathrm{~mm}$ continuum emission ${ }^{a}$.

\begin{tabular}{|c|c|c|c|c|c|c|c|c|c|c|c|}
\hline IRAS & RA & Dec. & $\begin{array}{c}V_{\mathrm{lsr}}^{b} \\
\left(\mathrm{~km} \mathrm{~s}^{-1}\right)\end{array}$ & $\begin{array}{c}\text { Distance }^{c} \\
(\mathrm{kpc})\end{array}$ & $\begin{array}{c}R_{\mathrm{GC}}^{c} \\
(\mathrm{kpc})\end{array}$ & ID & $\begin{array}{l}\text { Offset } \\
\left({ }^{\prime \prime}, "\right)\end{array}$ & $r_{\text {aspect }}$ & $\begin{array}{l}R_{\text {eff }} \\
(\mathrm{pc})\end{array}$ & $\begin{array}{c}S_{\text {peak }} \\
\left(\mathrm{Jy} \mathrm{beam}^{-1}\right)\end{array}$ & $\begin{array}{l}S_{\text {total }} \\
(\mathrm{Jy})\end{array}$ \\
\hline \multirow[t]{2}{*}{ I08076-3556 } & \multirow[t]{2}{*}{ 08:09:32.39 } & \multirow[t]{2}{*}{$-36: 05: 13.2$} & \multirow[t]{2}{*}{5.9} & \multirow[t]{2}{*}{0.4} & \multirow[t]{2}{*}{8.45} & 1 & $(8.56,15.49)$ & 1.37 & 0.01 & 0.03 & 0.03 \\
\hline & & & & & & 2 & $(4.83,-4.90)$ & 1.07 & 0.02 & 0.01 & 0.02 \\
\hline I08303-4303 & $08: 46: 32.90$ & $-43: 13: 54.0$ & 14.3 & 2.3 & 8.96 & 1 & $(3.87,7.99)$ & 1.50 & 0.10 & 0.06 & 0.10 \\
\hline \multirow[t]{2}{*}{ I08448-4343 } & \multirow[t]{2}{*}{ 08:48:47.07 } & \multirow[t]{2}{*}{$-43: 54: 35.9$} & \multirow[t]{2}{*}{3.7} & \multirow[t]{2}{*}{0.7} & \multirow[t]{2}{*}{8.43} & 1 & $(2.86,-0.25)$ & 3.86 & 0.05 & 0.04 & 0.09 \\
\hline & & & & & & 2 & $(21.53,7.53)$ & 1.87 & 0.05 & 0.03 & 0.09 \\
\hline I08470-4243 & 09:03:32.84 & $-42: 54: 31.0$ & 12 & 2.1 & 8.83 & 1 & $(8.15,4.60)$ & 2.10 & 0.09 & 0.07 & 0.12 \\
\hline I09002-4732 & 09:11:07.29 & $-47: 44: 00.8$ & 3.1 & 1.2 & 8.45 & 1 & $(0.32,-9.16)$ & 1.08 & 0.03 & 3.74 & 4.26 \\
\hline
\end{tabular}

${ }^{a}$ The full catalogue is available on line.

${ }^{b}$ The $V_{\text {lsr }}$ values are from Bronfman et al. (1996).

${ }^{c}$ The distances are from Urquhart et al. (2018) and Faúndez et al. (2004). 
Table A2. Selection of compact objects identified in $\mathrm{H}^{13} \mathrm{CO}^{+} J=1-0^{a}$.

\begin{tabular}{lccccccrrr}
\hline IRAS & ID & $\begin{array}{c}\text { Offset } \\
(",)^{\prime \prime}\end{array}$ & $r_{\text {aspect }}$ & $\begin{array}{c}R_{\text {eff }} \\
(\mathrm{pc})\end{array}$ & $\begin{array}{c}d_{\text {peak }} \\
(\mathrm{pc})\end{array}$ & $\begin{array}{c}S_{\text {peak }} \\
\left(\mathrm{Jy} \mathrm{beam}^{-1} \mathrm{~km} \mathrm{~s}^{-1}\right)\end{array}$ & $\begin{array}{c}S_{\text {total }} \\
\left(\mathrm{Jy} \mathrm{km} \mathrm{s}^{-1}\right)\end{array}$ & $\begin{array}{c}V_{\mathrm{cmp}} \\
\left(\mathrm{km} \mathrm{s}^{-1}\right)\end{array}$ & $\begin{array}{c}\sigma \\
\left(\mathrm{km} \mathrm{s}^{-1}\right)\end{array}$ \\
\hline I08076-3556 & 1 & $(7.26,10.15)$ & 3.39 & 0.06 & 0.01 & 3.12 & 16.97 & 6.21 & 0.28 \\
I08303-4303 & 1 & $(9.89,10.84)$ & 1.57 & 0.26 & 0.07 & 8.71 & 30.18 & 14.53 & 1.00 \\
I08448-4343 & 1 & $(12.76,1.15)$ & 3.16 & 0.09 & 0.03 & 5.37 & 21.38 & 2.78 & 0.84 \\
& 2 & $(30.80,15.93)$ & 1.77 & 0.07 & 0.11 & 3.62 & 10.55 & 5.06 & 0.66 \\
I08470-4243 & 1 & $(4.66,17.54)$ & 1.16 & 0.22 & 0.14 & 4.06 & 12.96 & 13.38 & 0.94 \\
& 2 & $(7.57,9.86)$ & 3.86 & 0.23 & 0.05 & 3.78 & 16.72 & 12.06 & 0.74 \\
I09002-4732 & 1 & $(19.98,19.19)$ & 1.70 & 0.10 & 0.20 & 8.48 & 17.89 & 2.44 & 1.08 \\
& 2 & $(12.54,-11.26)$ & 1.52 & 0.10 & 0.07 & 5.33 & 11.92 & 4.25 & 0.70 \\
& 3 & $(18.09,-15.62)$ & 2.74 & 0.09 & 0.11 & 4.33 & 9.84 & 2.54 & 0.62 \\
& 4 & $(1.97,-36.41)$ & 3.50 & 0.08 & 0.16 & 4.44 & 8.91 & 3.25 & 0.57 \\
\hline
\end{tabular}

${ }^{a}$ The full catalogue is available on line.

Table A3. Selection of compact objects identified in $\mathrm{H}^{13} \mathrm{CN} J=1-0^{a}$.

\begin{tabular}{lccccccr}
\hline IRAS & ID & $\begin{array}{c}\text { Offset } \\
\left(",,^{\prime \prime}\right.\end{array}$ & $r_{\text {aspect }}$ & $\begin{array}{c}R_{\text {eff }} \\
(\mathrm{pc})\end{array}$ & $\begin{array}{c}d_{\text {peak }} \\
(\mathrm{pc})\end{array}$ & $\begin{array}{c}S_{\text {peak }} \\
\left(\mathrm{Jy} \mathrm{beam}^{-1} \mathrm{~km} \mathrm{~s}^{-1}\right)\end{array}$ & $\begin{array}{c}S_{\text {total }} \\
\left(\mathrm{Jy} \mathrm{km} \mathrm{s}^{-1}\right)\end{array}$ \\
\hline I08076-3556 & 1 & $(9.76,14.74)$ & 1.34 & 0.03 & 0.00 & 2.79 & 6.29 \\
$\mathrm{I} 08303-4303$ & 1 & $(6.08,7.36)$ & 1.19 & 0.14 & 0.03 & 17.00 & 30.65 \\
I08448-4343 & 1 & $(1.27,0.58)$ & 1.63 & 0.08 & 0.01 & 10.10 & 33.88 \\
& 2 & $(6.53,-13.19)$ & 3.07 & 0.04 & 0.05 & 9.73 & 18.57 \\
I08470-4243 & 1 & $(8.30,5.30)$ & 3.36 & 0.15 & 0.01 & 13.50 & 33.76 \\
I09002-4732 & 1 & $(24.39,21.85)$ & 2.03 & 0.14 & 0.23 & 11.70 & 41.69 \\
& 2 & $(20.94,-12.69)$ & 4.59 & 0.08 & 0.12 & 4.46 & 9.71 \\
& 3 & $(13.92,-10.52)$ & 2.36 & 0.05 & 0.08 & 9.52 & 13.40 \\
& 4 & $(18.83,-23.57)$ & 1.27 & 0.10 & 0.14 & 6.35 & 14.80 \\
\hline
\end{tabular}

${ }^{a}$ The full catalogue is available on line.

Table A4. Selection of compact objects identified in $\mathrm{HCO}^{+} J=1-0^{a}$.

\begin{tabular}{|c|c|c|c|c|c|c|c|c|c|}
\hline IRAS & ID & $\begin{array}{l}\text { Offset } \\
\left(",{ }^{\prime \prime}\right)\end{array}$ & $\mathrm{r}_{\text {aspect }}$ & $\begin{array}{l}R_{\text {eff }} \\
(\mathrm{pc})\end{array}$ & $\begin{array}{l}d_{\text {peak }} \\
(\mathrm{pc})\end{array}$ & $\begin{array}{c}\mathrm{S}_{\text {peak }} \\
\left(\mathrm{Jy} \mathrm{beam}^{-1} \mathrm{~km} \mathrm{~s}^{-1}\right)\end{array}$ & $\begin{array}{c}\mathrm{S}_{\text {total }} \\
\left(\mathrm{Jy} \mathrm{km} \mathrm{s}^{-1}\right)\end{array}$ & $\begin{array}{c}\mathrm{V}_{\mathrm{cmp}} \\
\left(\mathrm{km} \mathrm{s}^{-1}\right)\end{array}$ & $\begin{array}{c}\sigma \\
\left(\mathrm{km} \mathrm{s}^{-1}\right)\end{array}$ \\
\hline \multicolumn{10}{|c|}{$\mathrm{HCO}^{+} \mathrm{J}=1-0$} \\
\hline I08076-3556 & 1 & $(10.31,13.96)$ & 1.18 & 0.04 & 0.00 & 21.80 & 57.20 & 6.46 & 1.06 \\
\hline I08303-4303 & 1 & $(11.51,6.91)$ & 2.05 & 0.34 & 0.09 & 38.30 & 182.70 & 13.93 & 2.05 \\
\hline \multirow[t]{2}{*}{ I08448-4343 } & 1 & $(4.88,1.10)$ & 1.53 & 0.09 & 0.01 & 59.80 & 247.60 & 2.82 & 1.92 \\
\hline & 2 & $(24.45,11.80)$ & 1.30 & 0.08 & 0.08 & 37.80 & 127.00 & 5.23 & 1.61 \\
\hline I08470-4243 & 1 & $(7.60,6.26)$ & 2.22 & 0.24 & 0.02 & 90.60 & 325.40 & 12.78 & 1.96 \\
\hline \multirow[t]{3}{*}{ I09002-4732 } & 1 & $(18.83,14.75)$ & 1.13 & 0.13 & 0.18 & 23.50 & 73.10 & -0.69 & 0.22 \\
\hline & 2 & $(3.11,-2.59)$ & 2.37 & 0.13 & 0.04 & 30.30 & 104.70 & 3.28 & 3.19 \\
\hline & 3 & $(14.81,-12.22)$ & 1.16 & 0.13 & 0.09 & 33.70 & 115.50 & 5.57 & 1.45 \\
\hline
\end{tabular}

${ }^{a}$ The full catalogue is available on line.

Table A5. Selection of compact objects identified in $\mathrm{HCN} J=1-0^{a}$.

\begin{tabular}{lccccccr}
\hline IRAS & ID & $\begin{array}{c}\text { Offset } \\
\left({ }^{\prime \prime},{ }^{\prime \prime}\right.\end{array}$ & $r_{\text {aspect }}$ & $\begin{array}{c}R_{\text {eff }} \\
(\mathrm{pc})\end{array}$ & $\begin{array}{c}d_{\text {peak }} \\
(\mathrm{pc})\end{array}$ & $\begin{array}{c}S_{\text {peak }} \\
\left(\mathrm{Jy} \mathrm{beam}^{-1} \mathrm{~km} \mathrm{~s}^{-1}\right)\end{array}$ & $\begin{array}{c}S_{\text {total }} \\
\left(\mathrm{Jy} \mathrm{km} \mathrm{s}^{-1}\right)\end{array}$ \\
\hline I08076-3556 & 1 & $(8.32,15.53)$ & 1.31 & 0.03 & 0.00 & 21.70 & 45.16 \\
$\mathrm{I} 08303-4303$ & 1 & $(7.49,8.29)$ & 1.25 & 0.19 & 0.04 & 78.50 & 181.90 \\
I08448-4343 & 1 & $(3.31,0.00)$ & 1.43 & 0.07 & 0.00 & 95.20 & 272.50 \\
& 2 & $(24.67,11.75)$ & 1.15 & 0.06 & 0.08 & 48.70 & 120.40 \\
I08470-4243 & 1 & $(8.56,3.96)$ & 2.07 & 0.17 & 0.01 & 158.00 & 404.00 \\
I09002-4732 & 1 & $(23.34,24.63)$ & 3.26 & 0.16 & 0.24 & 34.50 & 170.20 \\
& 2 & $(17.77,-10.99)$ & 2.25 & 0.13 & 0.10 & 46.40 & 155.70 \\
& 3 & $(12.29,-10.35)$ & 2.37 & 0.09 & 0.07 & 42.80 & 92.44 \\
& 4 & $(15.72,-27.64)$ & 1.73 & 0.17 & 0.14 & 32.70 & 162.70 \\
\hline
\end{tabular}

${ }^{a}$ The full catalogue is available on line. 
Table A6. Parameters of selection from 119 sources $^{a}$.

\begin{tabular}{|c|c|c|c|c|c|c|c|c|c|c|}
\hline IRAS & $\begin{array}{l}R_{\text {eff }} \\
\text { (pc) }\end{array}$ & $\begin{array}{l}\mathrm{T}_{d} \\
(\mathrm{~K})\end{array}$ & $\begin{array}{c}\log \left[\mathrm{L}_{\mathrm{bol}}\right] \\
\left(\mathrm{L}_{\odot}\right)\end{array}$ & $\begin{array}{c}\log \left[\mathrm{M}_{\text {clump }}\right] \\
\left(\mathrm{M}_{\odot}\right)\end{array}$ & $\begin{array}{c}\log \left[\mathrm{L}_{\mathrm{H}^{13} \mathrm{CO}^{+}}^{\prime}\right] \\
\left(\mathrm{Kkm} \mathrm{s}^{-1} \mathrm{pc}^{2}\right)\end{array}$ & $\begin{array}{c}\log \left[\mathrm{L}_{\mathrm{H}^{13} \mathrm{CN}^{\prime}}^{\prime}\right] \\
\left(\mathrm{Kkm} \mathrm{s}^{-1} \mathrm{pc}^{2}\right)\end{array}$ & $\begin{array}{c}\log \left[\mathrm{L}_{\mathrm{HCO}^{+}}^{\prime}\right] \\
\left(\mathrm{Kkm} \mathrm{s}^{-1} \mathrm{pc}^{2}\right)\end{array}$ & $\begin{array}{c}\log \left[\mathrm{L}_{\mathrm{HCN}}^{\prime}\right] \\
\left(\mathrm{Kkm} \mathrm{s}^{-1} \mathrm{pc}^{2}\right)\end{array}$ & $\begin{array}{c}\log \left[\mathrm{M}_{\text {vir }}^{13}\right] \\
\left(\mathrm{M}_{\odot}\right)\end{array}$ & $\begin{array}{c}\log \left[\mathrm{M}_{\mathrm{vir}}^{12}\right] \\
\left(\mathrm{M}_{\odot}\right)\end{array}$ \\
\hline I08303-4303 & 0.32 & 30 & 3.83 & 2.41 & -0.16 & -0.15 & 0.60 & 0.62 & 2.33 & 3.07 \\
\hline I08470-4243 & 0.32 & 33 & 4.04 & 2.36 & -0.25 & -0.19 & 0.77 & 0.89 & 2.44 & 2.88 \\
\hline I09002-4732 & 0.24 & 39 & 4.59 & 2.41 & -1.13 & -1.08 & -0.21 & -0.24 & 1.58 & 3.12 \\
\hline I09018-4816 & 0.44 & 31 & 4.72 & 2.99 & -0.21 & 0.11 & 0.80 & 0.82 & 2.43 & 3.09 \\
\hline
\end{tabular}

${ }^{a}$ The full catalogue is available on line.

${ }^{b} \mathrm{R}_{\text {eff }}, \mathrm{T}_{d}, \mathrm{~L}_{\text {bol }}$, and $\mathrm{M}_{\text {clump }}$ are compiled by Liu et al. (2020), which are adopted from Urquhart et al. (2018) and Faúndez et al. (2004).

${ }^{1}$ Shanghai Astronomical Observatory, Chinese Academy of Sciences, 80 Nandan Road, Shanghai 200030, People's Republic of China

${ }^{2}$ Korea Astronomy and Space Science Institute, 776 Daedeokdaero, Yuseong-gu, Daejeon 34055, Republic of Korea

${ }^{3}$ Key Laboratory for Research in Galaxies and Cosmology, Shanghai Astronomical Observatory, Chinese Academy of Sciences, 80 Nandan Road, Shanghai 200030, People's Republic of China

${ }^{4}$ Department of Astronomy, The University of Texas at Austin, 2515 Speedway, Stop C1400, Austin, TX 78712-1205, USA

${ }^{5}$ University of Science and Technology, Korea (UST), 217 Gajeong-ro, Yuseong-gu, Daejeon 34113, Republic of Korea

${ }^{6}$ Jet Propulsion Laboratory, California Institute of Technology, 4800 Oak Grove Drive, Pasadena, CA 91109, USA

${ }^{7}$ Institute of Astronomy and Astrophysics, Academia Sinica. $11 F$ of Astronomy-Mathematics Building, AS/NTU No. 1, Section 4, Roosevelt Rd., Taipei 10617, Taiwan

${ }^{8}$ Center for Astrophysics | Harvard \& Smithsonian, 60 Garden Street, Cambridge, MA 02138, USA

${ }^{9}$ National Astronomical Observatory of Japan, National Institutes of Natural Sciences, 2-21-1 Osawa, Mitaka, Tokyo 181-8588, Japan

${ }^{10}$ Kavli Institute for Astronomy and Astrophysics, Peking University, 5 Yiheyuan Road, Haidian District, Beijing 100871, People's Republic of China

${ }^{11}$ Department of Physics, University of Helsinki, PO Box 64, FI-00014, Finland

${ }^{12}$ Departamento de Astronomía, Universidad de Chile, Las Condes, 7591245 Santiago, Chile

${ }^{13}$ School of Physics, University of New South Wales, Sydney, NSW 2052, Australia

${ }^{14}$ School of Space Research, Kyung Hee University, Yongin-Si, Gyeonggi-Do 17104, Republic of Korea
${ }^{15}$ National Astronomical Observatories, Chinese Academy of Sciences, Beijing 100012, People's Republic of China

${ }^{16}$ University of Chinese Academy of Sciences, Beijing 100049, People's Republic of China

${ }^{17}$ NAOC-UKZN Computational Astrophysics Centre, University of KwaZulu-Natal, Durban 4000, South Africa

${ }^{18}$ Department of Astronomy, University of California, Berkeley, CA 94720 , USA

${ }^{19}$ Department of Astronomy, Yunnan University, and Key Laboratory of Astroparticle Physics of Yunnan Province, Kunming 650091, People's Republic of China

${ }^{20}$ IRAP, Université de Toulouse, CNRS, UPS, CNES, F-31400 Toulouse, France

${ }^{21}$ Indian Institute of Space Science and Technology, Thiruvananthapuram 695 547, Kerala, India

${ }^{22}$ Department of Astronomy, Eötvös Loránd University, Pázmány Péter sétány 1/A, H-1117 Budapest, Hungary

${ }^{23}$ Department of Astronomy, Peking University, 100871 Beijing, People's Republic of China

${ }^{24}$ Departamento de Astronomía, Universidad de Concepción, Av. Esteban Iturra s/n, Distrito Universitario 160-C, Chile

${ }^{25}$ College of Science, Yunnan Agricultural University, Kunming 650201, People's Republic of China

${ }^{26}$ Key Laboratory of Radio Astronomy, Chinese Academy of Sciences, Nanjing 210008, People's Republic of China

${ }^{27}$ School of Physics and Astronomy, Sun Yat-sen University, 2 Daxue Road, Zhuhai, Guangdong 519082, People's Republic of China

This paper has been typeset from a $\mathrm{T}_{\mathrm{E}} \mathrm{X} / \mathrm{L} \mathrm{T} \mathrm{T}_{\mathrm{E}} \mathrm{X}$ file prepared by the author. 